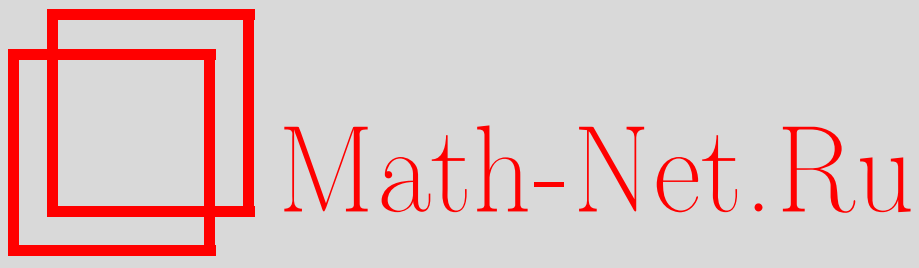

Г. М. Губреев, $L_{2}$-устойчивые полугруппы, веса Макенхаупта и безусловные базисы из значений квазиэкспонент, Матем. сб., 1999, том 190, номер 12, 3-36

DOI: https://doi.org/10.4213/sm442

Использование Общероссийского математического портала Math-Net.Ru подразумевает, что вы прочитали и согласны с пользовательским соглашением http://www . mathnet.ru/rus/agreement

Параметры загрузки:

IP: 54.80 .97 .219

26 апреля 2023 г., 13:08:12 


\title{
Г.М. Губреев
}

\section{$L_{2}$-устойчивые полугруппы, веса Макенхаупта и безусловные базисы из значений квазиэкспонент}

\begin{abstract}
В работе выделен класс неограниченных операторов с дискретным спектром, действующих в сепарабельном гильбертовом пространстве, для которых условие быть генератором $L_{2}$-устойчивой полугруппы равносильно подобию скалярной модели С.-Надя-Фойаша. При доказательстве этого результата установлена связь с теорией весов Макенхаупта. Вьводится также критерий подобия диссипативного одноклеточного оператора простейшему оператору интегрирования. Вводится понятие квазиэкспоненты - абстрактного аналога экспоненты. В качестве приложения дается описание всех безусловных базисов гильбертова пространства из значений квазиэкспонент.
\end{abstract}

Библиография: 17 названий.

\section{Введение}

Через $\Lambda^{(\exp )}$ обозначается [1] множество линейных ограниченных операторов $B$, действующих в сепарабельном гильбертовом пространстве $\mathfrak{H}$ и удовлетворяющих условиям:

1) $\sigma(B)=\{0\}$

2) $\frac{1}{2 i}\left(B-B^{*}\right) \geqslant 0$;

3) оператор-функция $(I-z B)^{-1}$ имеет конечньй экспоненциальный тип.

В дальнейшем всегда будем предполагать, что Ker $B=\{0\}$. Пару векторов $f, g \in \mathfrak{H}$ назовем согласованной с оператором $B$, если оператор

$$
K h:=B h+(h, f) g, \quad h \in \mathfrak{H},
$$

обладает свойством: $\operatorname{Ker} K=\operatorname{Ker} K^{*}=\{0\}$. Согласованность пары $f, g$ равносильна справедливости двух импликаций: если $g=B u$, то $(u, f) \neq-1$, и если $f=B^{*} v$, то $(v, g) \neq-1$. Пусть пара $f, g \in \mathfrak{H}$ согласована с оператором $B$. Тогда равенствами

$$
h=l+\left(B^{-1} l, f\right) g, \quad A h=B^{-1} l \quad \forall l \in \mathfrak{L}:=B \mathfrak{H}
$$

корректно определяется замкнутый неограниченный плотнозаданный в $\mathfrak{H}$ оператор $A$. Простые выкладки показывают, что

$$
\begin{gathered}
(A-z I)^{-1} h=B(I-z B)^{-1} h+\varphi^{-1}(z)\left((I-z B)^{-1} h, f\right) g(z), \quad h \in \mathfrak{H}, \\
g(z):=(I-z B)^{-1} g,
\end{gathered}
$$

Работа выполнена при частичной поддержке Правительства Украины и Фонда гражданских исследований США (грант UMI-298). 
где целая функция $\varphi$ экспоненциального типа имеет вид

$$
\varphi(z)=1-z(g(z), f)
$$

В дальнейшем экспоненциальный тип целой оператор-функции $(I-z B)^{-1}$ будем обозначать через $a<\infty$. Будем также считать, что

$$
h_{\varphi}(\pi / 2)=0, \quad h_{\varphi}(-\pi / 2)=a,
$$

где $h_{\varphi}-$ индикатор роста функции $\varphi$. Это естественное условие в интересующем нас случае необходимо для полноты в $\mathfrak{H}$ системы корневых векторов оператора $A$ (см. $\S 2)$. Кстати сказать, из (0.2) вытекает, что $\sigma(A)$ совпадает с последовательностью $\left\{z_{k}\right\}_{-\infty}^{+\infty}$ корней функции $\varphi$, а соответствующее корневое подпространство натягивается на векторы $g\left(z_{k}\right), g^{\prime}\left(z_{k}\right), \ldots, g^{\left(m_{k}-1\right)}\left(z_{k}\right)$, где $m_{k}-$ кратность корня $z_{k}$, причем $A g\left(z_{k}\right)=z_{k} g\left(z_{k}\right)$.

В настоящей работе излагается аналитический аппарат исследования спектральной структуры операторов $A$ вида (0.1), а также вольтерровых диссипативных операторов $B \in \Lambda^{(\exp )}$. Изложим кратко суть предлагаемого подхода. Отправным пунктом наших построений является задача об описания всех операторов $A$, которые генерируют $L_{2}$-устойчивые полугрупшы $U_{t}=\exp \{i A t\}, t>0$. Haпомним [2], что полугруппа $U_{t}$ класса $C_{0}$ назьвается $L_{2}$-yстойчивой, если $\left\|U_{t} h\right\| \in$ $L_{2}\left(\mathbb{R}_{+}\right)$для каждого $h \in \mathfrak{H}$. Конечно, можно было бы воспользоваться формальньм критерием принадлежности полугруппы классу $C_{0}$ (теорема Хилле-Филлипса-Феллера-Иосиды-Миядеры [2]), но вряд ли на этом пути можно получить точные результаты. Одним из основных достижений этой статьи является установление связи между задачей о полугруппах и теорией $A_{2}$-весов Макенхаупта на вешественной оси. Это дало возможность свести общую ситуацию к частному случаю $\mathfrak{H}=L_{2}(0, a) ; g$ каноническим образом строится по весу Макенхаупта, $B=J_{a}$, где

$$
\left(J_{a} h\right)(x)=i \int_{0}^{x} h(t) d t, \quad h \in L_{2}(0, a) .
$$

Такие операторы были предметом рассмотрения работ [3]-[5], где, в частности, были получены критерии $L_{2}$-устойчивости полугрупп. На основании результатов этих работ здесь строятся модели С.-Надя-Фойаша генераторов вида (0.1) $L_{2}$-устойчивых полугруп, изучаются их свойства, доказывается, что $U_{t} L_{2}$-устойчива лишь в случае подобия $B$ и оператора $J_{a}$. Тем самьм предложен новый подход к хорошо известной проблеме подобия вольтерровых операторов.

Результаты статьи вполне пригодны и для решения задачи о генераторах $C_{0}$-полугрупп. Дополнительное ограничение $L_{2}$-устойчивости введено лишь для удобства изложения и упрошения формулировок. Более подробно об этом сказано в конце $\S 3$.

Конкретным приложениям результатов $\S \S 1-3$ к теории канонических систем дифференциальных уравнений, к задачам подобия линейных операторов будет посвящена отдельная работа. Здесь мы рассмотрим лишш только приложение к теории безусловных базисов гильбертовых пространств, составленных из значений целых вектор-функций экспоненциального типа. 
В $\S 4$ излагается развитие некоторых результатов работы [6] и дается полное решение задачи из [7]. Чтобы сформулировать, о чем будет идти речь, дадим определение квазиэкспоненты - абстрактного аналога экспоненты.

Пусть $e: \mathbb{C} \rightarrow \mathfrak{H}$ - целая функция экспоненциального типа со значениями в сепарабельном пространстве $\mathfrak{H}$. Введем обозначение

$$
\tilde{e}(\lambda):=\lambda^{-1}(e(\lambda)-e(0)) .
$$

ОПРЕДЕЛЕНИЕ 1. Функция $е$ называется квазиэкспонентой, если

1) для каждого $z \in \mathbb{C}$ сушествует такой вектор $h_{z} \in \mathfrak{H}$, что

$$
\left.(\tilde{e}(\lambda), e(z))=\left(e(\lambda), h_{z}\right)\right) \quad \forall \lambda \in \mathbb{C} ;
$$

2) формы $\operatorname{Im}\left(\sum_{k} c_{k} \tilde{e}\left(\lambda_{k}\right), \sum_{k} c_{k} e\left(\lambda_{k}\right)\right) \geqslant 0$ для любых конечных совокупностей комплексных чисел $\left\{c_{k}\right\},\left\{\lambda_{k}\right\}$.

ОПРЕДЕЛЕНИЕ 2. Квазиэкспонента $е$ называется регулярной, если существует хотя бы одна такая последовательность $\left\{z_{k}\right\}_{-\infty}^{+\infty}\left(\inf _{k} \operatorname{Im} z_{k}>0\right)$, что семейство векторов $\left\{e\left(z_{k}\right)\right\}_{-\infty}^{+\infty}$ образует безусловный базис пространства $\mathfrak{H}$. Напомним, что в случае пространства Гильберта безусловный базис - это такой базис, который после нормировки становится базисом Рисса.

В $\S 4$ работы решены две задачи: 1) дано описание всех регулярных квазиэкспонент со значениями в $\mathfrak{H} ; 2$ ) для каждой регулярной квазиэкспоненты получено описание безусловных базисов пространства $\mathfrak{H}$, составленњых из ее значений.

Результаты статьи частично анонсированы в [8], [9].

\section{§ 1. $L_{2}$-устойчивые полугруппы и веса Макенхаупта}

Итак, пусть $A$ - оператор в пространстве $\mathfrak{H}$, определенный равенствами (0.1). Введем в рассмотрение вес $w^{2}$ на вешественной оси:

$$
w^{2}(x):=\|g(x)\|^{2}=\left\|(I-x B)^{-1} g\right\|^{2}, \quad x \in \mathbb{R} .
$$

Основньм результатом этого параграфа является

TЕОРема 1.1. Eсли оператор $A$ генерирует $L_{2}$-устойчивую полугруппу $U_{t}=\exp \{i A t\} \quad(t \geqslant 0)$, то вес $w^{2}$ удовлетворяет условию на $\mathbb{R}$

$$
\sup _{J}\left\{|J|^{-1} \int_{J} w^{2}(x) d x,|J|^{-1} \int_{J} w^{-2}(x) d x\right\}<\infty,
$$

где $J$ - произвольный интервал вещественной оси, $|J|$ - его длина.

Разобьем доказательство теоремы 1.1 на ряд лемм.

Лемма 1.1. Если оператор $A$ генерирует $L_{2}$-устойчивую полугруппу $U_{t}=$ $\exp \{i A t\}$, то вес $w^{2}$ удовлетворяет условиям

$$
\int_{\mathbb{R}} \frac{w^{ \pm 2}(x)}{1+x^{2}} d x<\infty
$$


ДокАЗАТЕЛЬСТво. $L_{2}$-устойчивая полугруппа $U_{t}$ в силу результата Датко [10] имеет отрицательный экспоненциальньй тип $\omega<0$. Поэтому область $\operatorname{Im} \lambda<-\omega$ свободна от точек спектра оператора $A$. Поскольку $U_{t}$ класса $C_{0}$, то имеет место интегральное представление резольвенты

$$
i(A-x I)^{-1} h=\int_{\mathbb{R}_{+}} \exp (-i x t) U_{t} h d t, \quad h \in \mathfrak{H}, \quad x \in \mathbb{R},
$$

из которого вытекает, что при каждом $h \in \mathfrak{H}$

$$
\int_{\mathbb{R}}\left\|(A-x I)^{-1} h\right\|^{2} d x=2 \pi \int_{\mathbb{R}_{+}}\left\|U_{t} h\right\|^{2} d t \leqslant 2 \pi \int_{\mathbb{R}_{+}}\left\|U_{t}\right\|^{2} d t\|h\|^{2} \leqslant K\|h\|^{2} .
$$

Чтобы извлечь из этой оценки полезную информацию, покажем сначала, что

$$
\int_{\mathbb{R}}\left\|B(I-x B)^{-1} h\right\|^{2} d x \leqslant M\|h\|^{2}, \quad h \in \mathfrak{H} .
$$

Для этого отметим, что неограниченный оператор $-i B^{-1}$, будучи диссипативным, генерирует сжимаюшую полугруппу $V_{t}:=\exp \left\{-i B^{-1} t\right\}(t \geqslant 0)$, которая в силу теоремы Винера-Пэли обращается в нуль при $t \geqslant a[2]$. Интегральное представление, аналогичное (1.2), дает

$$
i B(I-z B)^{-1} h=\int_{0}^{a} \exp \{i z t\} V_{t} h d t
$$

откуда и вытекает (1.4). Сразу же отметим, что с помощью аналогичных рассуждений можно получить и такую оценку:

$$
\int_{\mathbb{R}}\left\|B^{*}\left(I-x B^{*}\right)^{-1} h\right\|^{2} d x \leqslant M\|h\|^{2}, \quad h \in \mathfrak{H} .
$$

Наряду с $g(z)$ рассмотрим также целую вектор-функцию

$$
f(z):=\left(I-z B^{*}\right)^{-1} f, \quad z \in \mathbb{C}
$$

где вектор $f$ взят из формул (0.1). Из формулы Гильберта для резольвент вытекает, что

$$
(g(\lambda), f(\bar{\mu}))=(\varphi(\lambda)-\varphi(\mu))(\mu-\lambda)^{-1}, \quad \lambda, \mu \in \mathbb{C},
$$

где $\varphi$ определена равенством (0.3). Из соотношений $(0.2),(1.4),(1.5)$ выводим оценки

$$
\begin{aligned}
& \int_{\mathbb{R}}|\varphi(x)|^{-2}|(f(x), h)|^{2}\|g(x)\|^{2} d x \leqslant M_{1}\|h\|^{2}, \quad h \in \mathfrak{H} ; \\
& \int_{\mathbb{R}}|\varphi(x)|^{-2}|(g(x), h)|^{2}\|f(x)\|^{2} d x \leqslant M_{2}\|h\|^{2}, \quad h \in \mathfrak{H} .
\end{aligned}
$$


Полагая в первой оценке $h=g\left(z_{0}\right)$, где $z_{0} \in \sigma(A)$, с учетом (1.7) получим

$$
\int_{\mathbb{R}}\|g(x)\|^{2}\left|x-z_{0}\right|^{-2} d x=\int_{\mathbb{R}} w^{2}(x)\left|x-z_{0}\right|^{-2} d x<\infty .
$$

Чтобы закончить доказательство леммы, установим, что

$$
\|g(x)\|\|f(x)\||\varphi(x)|^{-1}>\frac{a}{2}, \quad x \in \mathbb{R}
$$

Функция $\varphi$ выражается через полугруппу $V_{t}=\exp \left\{-i B^{-1} t\right\}$ по формуле

$$
\begin{aligned}
\varphi(z) & =1-z(g(z), f)=1-z^{2}\left(B(I-z B)^{-1} g, f\right)-z(g, f) \\
& =1-z(g, f)+i z^{2} \int_{0}^{a} e^{i z t}\left(V_{t} g, f\right) d t
\end{aligned}
$$

Отсюда вытекает, что $\varphi$ принадлежит классу Картрайт и, стало быть, допускает представление

$$
\varphi(z)=e^{i \sigma z} \text { v.p. } \prod_{z_{k} \in \sigma(A)}\left(1-\frac{z}{z_{k}}\right), \quad \sigma \in \mathbb{R},
$$

в котором $\sigma=a / 2$, поскольку выполнено условие (0.4), а индикаторная диаграмма бесконечного произведения симметрична относительно начала координат [11]. Теперь оценка (1.10) получается следующим образом:

$$
\begin{aligned}
\|g(x)\|\|f(x)\|\left|\varphi^{-1}(x)\right| & \geqslant|(g(x), f(x))|\left|\varphi^{-1}(x)\right|=\left|\varphi^{\prime}(x)\right||\varphi(x)|^{-1} \\
& >\operatorname{Im}(\log \varphi(x))^{\prime}=\frac{a}{2}+\text { v.p. } \sum_{z_{k}} \operatorname{Im} z_{k}\left|x-z_{k}\right|^{-2}>\frac{a}{2} .
\end{aligned}
$$

Полагая в (1.9) $h=f(\bar{z})$, а также $h=f\left(\bar{z}_{0}\right), z_{0} \in \sigma(A)$, заключаем, что

$\int_{\mathbb{R}}\|f(x)\|^{2}|\varphi(x)|^{-2}|\varphi(x)-\varphi(z)|^{2}|x-z|^{-2} d x<\infty, \quad \int_{\mathbb{R}}\|f(x)\|^{2}\left|x-z_{0}\right|^{-2} d x<\infty$

и, следовательно,

$$
\int_{\mathbb{R}}\|f(x)\|^{2}|\varphi(x)|^{-2}\left|x-z_{0}\right|^{-2} d x<\infty
$$

С учетом (1.10) найдем

$$
\int_{\mathbb{R}} w^{-2}(x)\left|x-z_{0}\right|^{-2} d x \leqslant \frac{2}{a} \int_{\mathbb{R}}\|f(x)\|^{2}|\varphi(x)|^{-2}\left|x-z_{0}\right|^{-2} d x<\infty
$$

что и доказывает лемму.

Фактически нами попутно доказан еше один результат. 
Лемма 1.2. Если полугруппа $U_{t}=\exp \{i A t\} \quad L_{2}$-устойчива, то

$$
\int_{\mathbb{R}} w^{-2}(x)|(g(x), h)|^{2} d x \leqslant M\|h\|^{2}, \quad h \in \mathfrak{H} .
$$

Доказательство следует из (1.9), (1.10).

Из леммы 1.1 вытекает следующий важный для дальнейшего факт. Известно [12], что сходимость двух интегралов из формулировки леммы обеспечивает наличие внешних в смысле Берлинга функций $w_{ \pm}$в областях $\operatorname{Im} z>0, \operatorname{Im} z<0$ соответственно, которые обладают свойством

$$
\left|w_{+}(x+i 0)\right|^{2} \stackrel{\text { ח.в. }}{=} w^{2}(x), \quad\left|w_{-}(x-i 0)\right|^{2} \stackrel{\text { ח.в. }}{=} w^{2}(x), \quad x \in \mathbb{R} .
$$

ЛЕмма 1.3. В области $\operatorname{Im} z>0$ имеют место оценки

$$
\|g(z)\| \leqslant\left|w_{+}(z)\right|, \quad\|g(z)\| \leqslant C(\operatorname{Im} z)^{-1 / 2}\left|w_{+}(z)\right|
$$

с некоторой константой $C$.

ДокАЗАТЕЛЬСтво. Снова с помощью полугруппы $V_{t}$ для любого $h \in \mathfrak{H}$ вычислим

$$
(g(z), h)=z\left(B(I-z B)^{-1} g, h\right)+(g, h)=-i z \int_{0}^{a} e^{i z t}\left(V_{t} g, h\right) d t+(g, h) .
$$

Поэтому при любом $h \in \mathfrak{H}$ функции $\Phi(z):=(g(z), h)(z+i)^{-1}$ принадлежат классу Харди $H_{+}^{2}$. На вещественной оси имеем

$$
|\Phi(x)| \leqslant w(x)|x+i|^{-1}|| h \|, \quad \log |\Phi(x)|=\log \left|\Phi^{\text {out }}(x)\right| \leqslant \log \left(w(x)|x+i|^{-1}\|h\|\right),
$$

где $\Phi^{\text {out }}-$ внешняя часть функции $\Phi$. Учитывая свойства внешних функций, найдем

$$
\begin{aligned}
\log |\Phi(z)| & \leqslant \log \left|\Phi^{\text {out }}(z)\right|=\frac{\operatorname{Im} z}{\pi} \int_{\mathbb{R}} \log \left|\Phi^{\text {out }}(x)\right||x-z|^{-2} d x \\
& \leqslant \frac{\operatorname{Im} z}{\pi} \int_{\mathbb{R}} \log \left(w(x)|x+i|^{-1}\|h\|\right)|x-z|^{-2} d x \\
& =\log \left(\left|w_{+}(z)\right||x+i|^{-1}\|h\|\right), \quad \operatorname{Im} z>0 .
\end{aligned}
$$

Таким образом, $|(g(z), h)| \leqslant\left|w_{+}(z)\right||| h \|$, т.е. первая часть леммы доказана.

Теперь уже ограниченная в области $\operatorname{Im} z>0$ функция $F(z):=(g(z), h) w_{+}^{-1}(z)$ в силу леммы 1.2 принадлежит $L_{2}(\mathbb{R})$. Поэтому $F \in H_{+}^{2}$ и, стало быть, справедлива оценка

$$
\begin{aligned}
|F(z)| & \leqslant \frac{1}{2}(\operatorname{Im} z)^{-1 / 2}\left(\int_{\mathbb{R}}|F(x)|^{2} d x\right)^{1 / 2} \\
& =\frac{1}{2}(\operatorname{Im} z)^{-1 / 2}\left(\int_{\mathbb{R}} w^{-2}(x)|(g(x), h)|^{2} d x\right)^{1 / 2} \leqslant C(\operatorname{Im} z)^{-1 / 2}\|h\|,
\end{aligned}
$$

которая и завершает доказательство.

Следующий важњый этап доказательства теоремы 1.1 состоит в оценке интегралов Пуассона весов $w^{2}$. Прием, использованный при доказательстве следующей леммы, представляет, на наш взгляд, определенный интерес. 
Лемма 1.4. Пусть оператор А генерирует $L_{2}$-устойчивую полугруппу $U_{t}$. Тогда

$$
\frac{|\operatorname{Im} z|}{\pi} \int_{\mathbb{R}} w^{2}(x)|x-z|^{-2} d x \leqslant K\left|w_{-}(z)\right|^{2}, \quad \operatorname{Im} z<0 .
$$

ДокаЗАТЕЛЬСтво. Наряду с $A$ рассмотрим оператор $A_{z}$ с блуждающей точкой спектра $z(\operatorname{Im} z>0)$. Определим $A_{z}$ равенствами $(0.1)$, в которых вместо вектора $f$ надлежит взять $f_{z}=\frac{\bar{z}_{0}}{\bar{z}}\left(I-\bar{z} B^{*}\right)\left(I-\bar{z}_{0} B^{*}\right)^{-1} f$, где $z_{0}$-какая-нибудь точка спектра оператора $A$. Нетрудно проверить, что новая пара $f_{z}, g$ согласована с оператором $B$. Пусть $\varphi_{z}$ - целая функция вида (0.3), отвечаюшая оператору $A_{z}$. Простая выкладка показывает, что

$$
\varphi_{z}(\lambda)=\frac{z_{0}}{z}(\lambda-z)\left(\lambda-z_{0}\right)^{-1} \varphi(\lambda) .
$$

Таким образом, $\sigma\left(A_{z}\right)=\left(\sigma(A) \backslash\left\{z_{0}\right\}\right) \cup\{z\}$ (в случае кратного корня $z_{0}$ эта запись означает, что кратность уменьшилась на единицу). Поскольку $\left(A_{z}-\lambda I\right)^{-1} g(z)=$ $(z-\lambda)^{-1} g(z)$, то

$$
\int_{\mathbb{R}}\left\|\left(A_{z}-x I\right)^{-1} g(z)\right\|^{2} d x=\pi(\operatorname{Im} z)^{-1}\|g(z)\|^{2} .
$$

Учитывая оценку (1.4) и формулу $(0.2)$, получим

$$
\int_{\mathbb{R}}\|g(x)\|^{2}\left|(I-x B)^{-1} g(z), f_{z}\right|^{2}\left|\varphi_{z}(x)\right|^{-2} d x \leqslant \pi(\operatorname{Im} z)^{-1}\|g(z)\|^{2}+M\|g(z)\|^{2} .
$$

Из (1.7) выводим, что

$$
\left((I-x B)^{-1} g(z), f_{z}\right) \varphi_{z}^{-1}(x)=(z-x)^{-1},
$$

поскольку $\varphi_{z}(z)=0$. Поэтому последнее неравенство перепишем в виде

$$
\int_{\mathbb{R}} w^{2}(x)|x-z|^{-2} d x \leqslant(\pi+M \operatorname{Im} z)(\operatorname{Im} z)^{-1}\|g(z)\|^{2}, \quad \operatorname{Im} z>0 .
$$

Например, если $\operatorname{Im} z>\delta>0$, то, воспользовавшись второй оценкой из леммы 1.3 , получим

$$
\int_{\mathbb{R}} w^{2}(x)|x-z|^{-2} d x \leqslant M_{1}\|g(z)\|^{2}<M_{2}(\operatorname{Im} z)^{-1}\left|w_{+}(z)\right|^{2} .
$$

При малых $\operatorname{Im} z$ надлежит использовать первую оценку $\|g(z)\|$. Теперь уже просто выводится требуемое неравенство для интеграла Пуассона в области $\operatorname{Im} z<0$, поскольку функции $w_{ \pm}$, осуществляющие продолжение $w^{2}$ в верхнюю и нижнюю полуплоскости, связаны соотношением $w_{+}(z)=\overline{w_{-}(\bar{z})}$.

Перейдем теперь к формулировке двойственных результатов. Нетрудно видеть, что оператор $-A^{*}$ задается формулами

$$
h=m+\left(B_{1}^{-1} m, g_{1}\right) f, \quad-A^{*} h=B_{1}^{-1} m \quad \forall m \in \mathfrak{M}:=B_{1} \mathfrak{H},
$$

где $g_{1}=-g, B_{1}=-B^{*} \in \Lambda^{(\exp )}$. Из упоминаемого во введении критерия принадлежности классу $C_{0}$ вытекает, что полугруппа $V_{t}:=\exp \left\{-i A^{*} t\right\}(t>0) L_{2}$-устойчива и, стало быть, к оператору $-A^{*}$ применимы все предыдущие результаты, поскольку условие (0.4) автоматически выполняется. Введем в рассмотрение новьй вес

$$
W^{2}(x):=|\varphi(x)|^{2} w^{-2}(x), \quad x \in \mathbb{R} .
$$


ЛЕмМа 1.5. Если полугруппа $U_{t}=\exp \{i A t\} L_{2}$-устойчива, то

$$
\frac{|\operatorname{Im} z|}{\pi} \int_{\mathbb{R}} W^{2}(x)|x-z|^{-2} d x \leqslant M\left|W_{-}(z)\right|^{2}, \quad \operatorname{Im} z<0
$$

причем $W_{-}(z)=\varphi(z) w_{-}^{-1}(z) e^{-i a z}, \operatorname{Im} z<0$.

ДокАЗАТЕЛЬство. Как уже отмечалось, справедлив аналог леммы 1.4 для веса $w_{1}^{2}(x):=\left\|\left(I-x B_{1}\right)^{-1} f\right\|^{2}=\left\|\left(I+x B^{*}\right)^{-1} f\right\|^{2}, x \in \mathbb{R}$. Покажем, что

$$
\Delta \geqslant w_{1}(-x) w(x)|\varphi(x)|^{-1} \geqslant \delta>0, \quad x \in \mathbb{R}
$$

с некоторьми константами $\Delta, \delta$. В силу (1.10) достаточно доказать лишь оценку сверху. Из (1.2) вытекает, что $\left\|(A-x I)^{-1}\right\| \leqslant K_{1} \quad(x \in \mathbb{R})$. Поскольку $\left\|B(I-x B)^{-1}\right\| \leqslant K_{2}$ при вещественных $x$, то из $(0.2)$ выводим

$$
|\varphi(x)|^{-1}\left\|\left(I-x B^{*}\right)^{-1} f\right\|\left\|(I-x B)^{-1} g\right\| \leqslant \Delta,
$$

что и требовалось. Поэтому лемма 1.4 справедлива для веса $W^{2}(-x)$ и, стало быть, для веса $W^{2}$. Осталось доказать, что

$$
W_{-}(z)=\varphi(z) e^{-i a z} w_{-}^{-1}(z), \quad \operatorname{Im} z<0
$$

В самом деле, из интегрального представления функции $\varphi$, которое было получено при доказательстве леммы 1.1, заключаем, что целая функция

$$
\varphi(z)\left(z-z_{1}\right)^{-1}\left(z-z_{2}\right)^{-1} \quad\left(z_{1}, z_{2} \in \sigma(A)\right)
$$

принадлежит $L_{2}$ на вешественной оси. Из теоремы Винера-Пэли и $(0.4)$ вытекает, что

$$
f(z):=e^{-i a z} \varphi(z)\left(z-z_{1}\right)^{-1}\left(z-z_{2}\right)^{-1} \in H_{-}^{2}
$$

и поэтому допускает внутренне-внешнюю факторизацию. Функция $f$ не имеет корней в области $\operatorname{Im} z<0$. Следовательно, внутренний множитель сингулярен, а так как функция $f$ целая, то носитель представляющей меры сосредоточен в бесконечности [12]. Другим словами, внутренний множитель равен $\exp \{i z \gamma\}, \gamma \leqslant 0$. Но $\gamma=0$ в силу условия $h_{\varphi}(-\pi / 2)=a$. Следовательно, $W_{-}-$внешняя функция в области $\operatorname{Im} z<0$, так как она является отношением внешних функций $f$ и $w_{-}(z)\left(z-z_{1}\right)^{-1}\left(z-z_{2}\right)^{-1}$.

Наконец, докажем еще одну лемму, в формулировке которой

$$
(\mathscr{H} f)(x)=\frac{1}{\pi} \int_{\mathbb{R}} \frac{f(t) d t}{x-t}, \quad f \in L_{2}(\mathbb{R}),
$$

а через $g \mathscr{H}(\mathscr{H} g)$ обозначается произведение $\mathscr{H}$ и оператора умножения на функцию $g$. 
Лемма 1.6. Если оператора $A$ генерирует $L_{2}$-устойчивую полугруппу $U_{t}=$ $\exp \{i A t\}$, mo onepamop

$$
w \mathscr{H} w^{-1}-w \varphi^{-1} \mathscr{H} \varphi w^{-1}
$$

ограничен в $L_{2}(\mathbb{R})$.

ДокАЗАТЕЛЬСТво. Интеграл

$$
h=\int_{\mathbb{R}} g(x) p(x) d x \in \mathfrak{H}, \quad g(x)=(I-x B)^{-1} g,
$$

имеет смысл для каждой финитной непрерывной комплекснозначной функции $p$. Лемма 1.2 позволяет получить оценку

$$
\begin{aligned}
\left|\left(\int g(x) p(x) d x, l\right)\right|^{2} & =\left|\int(g(x), l) w^{-1}(x) p(x) w(x) d x\right|^{2} \\
& \leqslant \int|(g(x), l)|^{2} w^{-2}(x) d x \int|p(x)|^{2} w^{2}(x) d x \\
& \leqslant M\|l\|^{2} \int|p(x)|^{2} d x
\end{aligned}
$$

для каждого $l \in \mathfrak{H}$. Поэтому

$$
\left\|\int_{\mathbb{R}} g(x) p(x) d x\right\|^{2} \leqslant M\|l\|^{2} \int_{\mathbb{R}}|p(x)|^{2} w^{2}(x) d x
$$

и, следовательно, соответствие $p \rightarrow h$ продолжается до непрерывного отображения $L_{2}$ с мерой $w^{2}(x) d x$ в $\mathfrak{H}$.

Принимая во внимание (1.7), вычислим $(h, f(x))$, где

$$
h=\int_{\mathbb{R}} g(s) p(s) d s, \quad \int_{\mathbb{R}}|p(s)|^{2} w^{2}(s) d s<\infty .
$$

Имеем

$$
(h, f(x))=\int_{\mathbb{R}}(g(s), f(s)) p(s) d s=\int_{\mathbb{R}}(\varphi(s)-\varphi(x))(x-s)^{-1} p(s) d s .
$$

При доказательстве леммы 1.1 была получена оценка (1.8). Рассмотрим ее на векторе $h$, задаваемом равенством (1.15). Учитывая предыдущее равенство, а также (1.14), получим

$$
\begin{gathered}
\int_{\mathbb{R}} w^{2}(x)\left|\varphi^{-1}(x) \int_{\mathbb{R}}(\varphi(s)-\varphi(x))(x-s)^{-1} p(s) d s\right|^{2} d x \\
\quad=\int_{\mathbb{R}} w^{2}(x)\left|(\mathscr{H} p)(x)-\varphi^{-1}(x)(\mathscr{H} \varphi p)(x)\right|^{2} d x \\
\leqslant M_{1}\|h\|^{2} \leqslant M_{2} \int_{\mathbb{R}}|p(s)|^{2} w^{2}(s) d s .
\end{gathered}
$$

Полагая $q(s)=p(s) w(s)$, последнее неравенство перепишем в виде

$$
\left\|w \mathscr{H} w^{-1} q-w \varphi^{-1} \mathscr{H} \varphi w^{-1} q\right\|_{L_{2}(\mathbb{R})}^{2} \leqslant M_{2}\|q\|_{L_{2}(\mathbb{R})}^{2} .
$$


ДокАЗАТЕЛЬСТво тЕОРЕмЫ 1.1. Докажем, что каждое слагаемое в (1.13) является ограниченным оператором в $L_{2}(\mathbb{R})$. В силу $(1.12)$ вместе с оператором (1.13) ограниченным в $L_{2}(\mathbb{R})$ является также оператор $e^{i a x} w_{-} \mathscr{H} w_{-}^{-1} e^{-i a x}-$ $W_{-}^{-1} \mathscr{H} W_{-}$, где под $w_{-}(x), W_{-}(x)$ понимаются граничные значения $w_{-}(x-i 0)$, $W_{-}(x-i 0), x \in \mathbb{R}$. Поскольку $i \mathscr{H}=I-2 \mathbb{P}_{-}$, где $\mathbb{P}_{-}-$ортопроектор из $L_{2}(\mathbb{R})$ на $H_{-}^{2}$, то приходим к ограниченности в $L_{2}(\mathbb{R})$ оператора $e^{i a x} w_{-} \mathbb{P}_{-} w_{-}^{-1} e^{-i a x}-$ $W_{-}^{-1} \mathbb{P}_{-} W_{-}$. Если записать это на функциях $(x-z)^{-1}(\operatorname{Im} z<0)$, то получим

$\left\|e^{i a x} w_{-} \mathbb{P}_{-} w_{-}^{-1} e^{-i a x}(x-z)^{-1}-W_{-}^{-1} \mathbb{P}_{-} W_{-}(x-z)^{-1}\right\|_{L_{2}(\mathbb{R})} \leqslant K\left\|(x-z)^{-1}\right\|_{L_{2}(\mathbb{R})}$.

Легко видеть, что

$$
\begin{gathered}
\mathbb{P}_{-}\left(e^{-i a x} w_{-}^{-1}(x)(x-z)^{-1}\right)=\left(w_{-}^{-1}(x) e^{-i a x}-w_{-}^{-1}(z) e^{-i a z}\right)(x-z)^{-1} \\
\mathbb{P}_{-}\left(W_{-}(x)(x-z)^{-1}\right)=\left(W_{-}(x)-W_{-}(z)\right)(x-z)^{-1}, \quad \operatorname{Im} z<0 .
\end{gathered}
$$

Поэтому последнее неравенство перепишем в виде

$$
\left\|\frac{e^{i a x} w_{-}(x) w_{-}^{-1}(z) e^{-i a z}}{x-z}-\frac{W_{-}^{-1}(x) W_{-}(z)}{x-z}\right\|_{L_{2}(\mathbb{R})} \leqslant K_{1} \frac{1}{\sqrt{|\operatorname{Im} z|}}, \quad \operatorname{Im} z<0 .
$$

Таким образом,

$$
\begin{aligned}
& \left\|W^{-1}(x)(x-z)^{-1}\right\|_{L_{2}(\mathbb{R})}\left|W_{-}(z)\right| \\
& \quad \leqslant K_{1}|\operatorname{Im} z|^{-1 / 2}+\left\|w_{-}(x)(x-z)^{-1}\right\|_{L_{2}(\mathbb{R})}\left|w_{-}^{-1}(z)\right|\left|e^{i a z}\right| .
\end{aligned}
$$

Из леммы 1.4 выводим, что

$$
\left\|w_{-}(x)(x-z)^{-1}\right\|_{L_{2}(\mathbb{R})}\left|w_{-}^{-1}(z)\right|\left|e^{-i a z}\right| \leqslant K_{2}|\operatorname{Im} z|^{-1 / 2}, \quad \operatorname{Im} z<0 .
$$

Из двух последних неравенств получаем оценку интеграла Пуассона веса $W^{-2}$ :

$$
\frac{|\operatorname{Im} z|}{\pi} \int_{\mathbb{R}} W^{-2}(x)|x-z|^{-2} d x \leqslant K_{3}\left|W_{-}(z)\right|^{-2}, \quad \operatorname{Im} z<0 .
$$

С другой стороны, в лемме 1.5 получена аналогичная оценка для веса $W^{2}$. В [13] показано, что совокупность этих оценок равносильна тому, что вес $W^{2}$ удовлетворяет условию $\left(\mathrm{A}_{2}\right)$ на $\mathbb{R}$. Но тогда оператор $W_{-}^{-1} \mathscr{H} W_{-}$ограничен в $L_{2}(\mathbb{R})$ и, стало быть, таковым является и оператор $w_{-} \mathscr{H} w_{-}^{-1}$, т.е. веса $w^{2}, W^{2}$ удовлетворяют условию Макенхаупта.

СлЕДСтвиЕ. Если полугруппа $U_{t} \quad L_{2}$-устойчива, то веса $W^{2}(x)=$ $|\varphi(x)|^{2} w^{-2}(x), \quad w_{*}^{2}(x):=\left\|\left(I-x B^{*}\right)^{-1} f\right\|^{2}$ также удовлетворяют условию Макенхаупта на $\mathbb{R}$.

Действительно, при доказательстве леммы 1.5 была получена оценка $\Delta \geqslant$ $w_{*}(x) W^{-1}(x) \geqslant \delta, x \in \mathbb{R}$. 


\section{§ 2. Полнота собственных и присоединенных векторов}

Напомним, что экспоненциальный тип $(I-z B)^{-1}$ равен $a$.

Tеорема 2.1. Eсли полугруппа $U_{t}=\exp \{i A t\}, t>0, L_{2}$-устойчива, то семейство корневых векторов оператора $A$ полно в $\mathfrak{H}$ тогда и только тогда, когда выполнены условия (0.4).

ЛЕмма 2.1. Если выполнено условие теоремы 2.1, то g-ииклический вектор $B$, а $f$-ииклический вектор $B^{*}$, т.е.

$$
\mathfrak{H}=\operatorname{clos} \operatorname{span}\left\{B^{n} g\right\}=\operatorname{clos} \operatorname{span}\left\{\left(B^{*}\right)^{n} f\right\}, \quad n \in \mathbb{Z}, \quad n \geqslant 0 .
$$

ДокАЗАТЕЛЬство. Если $g$ не циклический, то через $\mathfrak{H}_{0}$ обозначим нетривиальное подпространство векторов $h_{0}$, для которых $\left((I-z B)^{-1} g, h_{0}\right) \equiv 0$, $z \in \mathbb{C}$. Подпространство $\mathfrak{H}_{0}$ инвариантно относительно $B^{*}$. Из $(0.2)$ вытекает, что $\left(A^{*}-z I\right)^{-1} \mid \mathfrak{H}_{0}=\left(\left(B_{0}^{*}\right)^{-1}-z I\right)^{-1}$, где $B_{0}^{*}=B^{*} \mid \mathfrak{H}_{0}$. Опеpaтор $\left(B_{0}^{*}\right)^{-1}$ сушествует, поскольку $\operatorname{Ker} B_{0}=\operatorname{Ker} B_{0}^{*}=\{0\}$, что является следствием условия $\operatorname{Ker} B=\{0\}$ (см. введение). Поэтому полугруппа $V_{-}(t):=U_{t}^{*} \mid \mathfrak{H}_{0}=\exp \left\{-i\left(B_{0}^{*}\right)^{-1} t\right\}, t>0, L_{2}$-устойчива. $\mathrm{C}$ другой стороны, неограниченный оператор $\left(B_{0}^{*}\right)^{-1}$, будучи диссипативным, генерирует сжимающую полугруппу $V_{+}(t):=\exp \left\{i\left(B_{0}^{*}\right)^{-1} t\right\}, t>0$. Таким образом, $V(t):=\exp \left\{i\left(B_{0}^{*}\right)^{-1} t\right\}, t \in \mathbb{R}$, является группой ограниченных в $\mathfrak{H}_{0}$ операторов. Последнее невозможно, поскольку из теоремы Винера-Пэли следует, что $V_{+}$ нильпотентна, т.е. $V_{+}(t)=0$ при $t>a$.

Совершенно аналогично доказывается цикличность $f$.

Из доказанной леммы следует, что $B$ одноклеточен, имеет ядерную мнимую часть $\operatorname{Im} B$ и $a=2 \mathrm{Sp} \operatorname{Im} B[1]$.

ДОКАЗАТЕЛЬСТво ТЕОРЕмЫ 2.1. Необходимость. Пусть семейство корневых подпространств полно в $\mathfrak{H}$. Тогда $\operatorname{Sp} \operatorname{Im} A^{-1}=\sum_{z_{k} \in \sigma(A)} \operatorname{Im}\left(z_{k}^{-1}\right)$ [14]. С другой стороны, принимая во внимание (1.7) и (0.2), при $z=0$ получим

$$
\operatorname{Sp} \operatorname{Im} A^{-1}=\operatorname{Sp} \operatorname{Im} B+\operatorname{Im}(g, f)=\frac{a}{2}-\left.\operatorname{Im}(\log \varphi(x))^{\prime}\right|_{x=0}
$$

При доказательстве леммы 1.1 установлено, что $\varphi$ класса Картрайт, и, следовательно,

$$
\varphi(z)=e^{i d z} \prod_{z_{k} \in \sigma(A)}\left(1-\frac{z}{z_{k}}\right), \quad d \in \mathbb{R} .
$$

Поэтому $\left.\operatorname{Im}(\log \varphi(x))^{\prime}\right|_{x=0}=d-\sum_{z_{k} \in \sigma(A)} \operatorname{Im}\left(z_{k}\right)^{-1}$ и из (2.1) заключаем, что $d=a / 2$. Поскольку индикаторная диаграмма бесконечного произведения в $(2.2)$ симметрична относительно вещественной оси, то $h_{\varphi}(\pi / 2)=-a / 2+\varkappa / 2$, $h_{\varphi}(-\pi / 2)=a / 2+\varkappa / 2$, где $\varkappa-$ ширина этой диаграммы [11]. Таким образом, достаточно доказать равенство $h_{\varphi}(-\pi / 2)=a$, которое равносильно тому, что экспоненциальный тип $\varphi$ равен $a$. Пусть он равен $\sigma<a$. Тогда тип $(g(z), f)$ не 
превосходит $\sigma$ и, если учесть равенство $B g(z)=z^{-1}(g(z)-g(0))$, то заключаем, что типы функций

$$
\left(B(I-z B)^{-1} B^{k} g,\left(B^{*}\right)^{j} f\right), \quad k, j \in \mathbb{Z}, \quad k, j \geqslant 0
$$

также не превосходят $\sigma$. Из леммы 2.1 вытекает, что этим же свойством обладают типы функций $\left(B(I-z B)^{-1} x, y\right)$, где $x, y$ пробегают плотные в $\mathfrak{H}$ линеалы. И наконец, из теоремы Винера-Пэли и оценки (см. (1.4))

$$
\int_{\mathbb{R}}\left|\left(B(I-x B)^{-1} p, l\right)\right|^{2} d x \leqslant M\|p\|^{2}\|l\|^{2}, \quad p, l \in \mathfrak{H},
$$

следует, что типы $\left(B(I-x B)^{-1} x, y\right)$ не превосходя $\sigma$ уже для всех $x, y \in \mathfrak{H}$. Последнее противоречит тому, что экспоненциальньй тип $(I-z B)^{-1}$ равен $a$.

Достаточность. В случае неполноты системы корневых векторов существует такой вектор $h_{0} \neq 0$, что функция

$$
G(z):=F(z) \varphi^{-1}(z), \quad F(z):=\left(g(z), h_{0}\right),
$$

целая не выше первого порядка роста. Учитывая неравенство $\|f\| \leqslant(1+|x|\|B\|) \times$ $\|f(x)\|, x \in \mathbb{R}$, и рассматривая оценку (1.9) на векторе $h_{0}$, получим

$$
\|f\|^{2} \int_{\mathbb{R}}|G(x)|^{2}(1+|x|\|B\|)^{-2} d x \leqslant \int_{\mathbb{R}}|G(x)|^{2}\|f(x)\|^{2} d x \leqslant M_{2}\left\|h_{0}\right\|^{2} .
$$

Поэтому целая функция $G(z)(z-\mu)^{-1}$, где $G(\mu)=0$, принадлежит $L_{2}(\mathbb{R})$ (случай отсутствия корней функции $G$ тривиален). Так как $\varphi, F$ - функции вполне регулярного роста, то из $(2.3)$ и (0.4) вытекает, что $h_{G}( \pm \pi / 2)=h_{F}( \pm \pi / 2)-h_{\varphi}( \pm \pi / 2) \leqslant 0$. Следовательно, $G(z) \equiv 0$ и из леммы 2.1 выводим, что $h_{0}=0$.

\section{§3. $L_{2}$-устойчивые полугруппы и подобие линейных операторов}

Пусть спектр оператора $A$ вида (0.1) совпадает с последовательностью $\left\{z_{k}\right\}_{-\infty}^{+\infty}$, $m_{k}$ - кратность собственного числа $z_{k}$. Как уже отмечалось, в случае $L_{2}$-устойчивости $U_{t}$ спектр $\sigma(A)$ лежит в некоторой области $\operatorname{Im} z>\delta>0$. Построим простейший диссипативный оператор с тем же спектром. Для этого рассмотрим в классе Харди $H_{-}^{2}$ семейство рациональных дробей

$$
r_{k}^{j}(z):=j !\left(z-z_{k}\right)^{-(j+1)}, \quad 0 \leqslant j<m_{k}, \quad z_{k} \in \sigma(A)
$$

Линейная замкнутая оболочка $K_{B}$ этого семейства в $H_{-}^{2}$ совпадает с ортогональным дополнением к подпространству $B H_{-}^{2}$, т.е. $K_{B}=H_{-}^{2} \ominus B H_{-}^{2}$, где $B$ - произведение Бляшке с множеством корней $\left\{z_{k}\right\}_{-\infty}^{+\infty}$ с учетом кратностей. Заметим, что произведение Бляшке всегда сходится, поскольку $\sigma(A)$ совпадает с множеством корней целой функции класса Картрайт [11]. Теперь в пространстве $K_{B}$ зададим модельный диссипативный оператор С.-Надя-Фойаша формулой

$$
\left(A_{m} f\right)(z)=z f(z)-\lim _{y \rightarrow-\infty} i y f(i y)
$$


отнеся к области определения $A_{m}$ все функции $f \in K_{B}$, для которых правая часть в $(3.2)$ принадлежит $K_{B}$. Тогда $\sigma\left(A_{m}\right)=\sigma(A)$, собственным вектором $A_{m}$ будет функция $r_{k}^{0}(z)=\left(z-z_{k}\right)^{-1}$, а остальные функции (3.1) являются корневыми, отвечающими точке $z_{k}$. Для случая сжатий с одномерным дефектом аналогичные результаты изложены в [15]. Сформулированные факты легко получаются с помощью соответствующего преобразования Кэли [3] или могут быть проверены непосредственно.

Через $L_{2}\left(\mathbb{R}, w^{2}\right)$ будем обозначать пространство $L_{2}(\mathbb{R})$ с мерой $w^{2}(x) d x$. Если $w^{2}$ удовлетворяет условию $\left(\mathrm{A}_{2}\right)$, то через $H_{-}^{2}\left(w^{2}\right)$ обозначим весовой класс Харди, состоящий из аналитических в области $\operatorname{Im} z<0$ функций $f$, для которых $f(z) w_{-}(z) \in H_{-}^{2}$ с нормой $\|f\|:=\left\|f w_{-}\right\|_{H_{-}^{2}}^{2}$. Классы $H_{-}^{2}\left(w^{2}\right)$ обладают многими свойствами безвесовых классов Харди [4]. В частности, $H_{-}^{2}\left(w^{2}\right)$ естественньм образом отождествляется с подпространством в $L_{2}\left(\mathbb{R}, w^{2}\right)$.

TеОрема 3.1. Oператор $A$ генерирует $L_{2}$-устойчивую полугруппу $U_{t}=$ $\exp \{i A t\}$ тогда и только тогда, когда:

1) $\sigma(A)$ лежсит в некоторой области $\operatorname{Im} z>\delta>0$;

2) операторьи $A$ u $A_{m}$ подобньь, m.е. $A_{m}=Q A Q^{-1}$.

При этом оператор $Q$ имеет вид

$$
(Q h)(z)=\mathbb{P}_{-}\left(w_{+}(x+i 0) \varphi^{-1}(x)\left((I-x B)^{-1} h, f\right)\right), \quad h \in \mathfrak{H}, \quad \operatorname{Im} z<0,
$$

әде $\mathbb{P}_{-}$- ортопроектор из $L_{2}(\mathbb{R})$ на $H_{-}^{2}$.

ДокАЗАТЕЛЬСтво. Необходимость. Из оценки (1.8) следует, что оператор

$$
(R h)(x):=\varphi^{-1}(x)\left((I-x B)^{-1} h, f\right), \quad h \in \mathfrak{H},
$$

непрерывно действует из $\mathfrak{H}$ в пространство $L_{2}\left(\mathbb{R}, w^{2}\right)$. Вычислим образ этого оператора. Если ввести обозначение

$$
g_{k}^{j}:=g^{(j)}\left(z_{k}\right), \quad 0 \leqslant j<m_{k}, \quad z_{k} \in \sigma(A),
$$

то в силу (1.7) найдем

$$
\begin{aligned}
\left(R g_{k}^{j}\right)(x) & =\varphi^{-1}(x)\left(g^{(j)}\left(z_{k}\right), f(x)\right)=\left.\varphi^{-1}(x) \frac{d^{j}}{d \lambda^{j}}\left\{(\varphi(\lambda)-\varphi(x))(x-\lambda)^{-1}\right\}\right|_{\lambda=z_{k}} \\
& =-j !\left(x-z_{k}\right)^{-(j+1)}, \quad 0 \leqslant j<m_{k}
\end{aligned}
$$

поскольку $\varphi\left(z_{k}\right)=\varphi^{\prime}\left(z_{k}\right)=\cdots=\varphi^{\left(m_{k}-1\right)}\left(z_{k}\right)=0, \varphi^{\left(m_{k}\right)}\left(z_{k}\right) \neq 0$. Далее, в силу лемм $1.2,1.3$ имеем $(g(z), h) w_{+}^{-1}(z) \in H_{+}^{2}$ для каждого $h \in \mathfrak{H}$. Поэтому для любой конечной суммы $\sum_{j, k} c_{k}^{j} g_{k}^{j}$ корневых векторов и для любого $h \in \mathfrak{H}$ получим оценку

$$
\begin{aligned}
\left|\left(\sum c_{k}^{j} g_{k}^{j}, h\right)\right|^{2} & =\left|\frac{1}{2 \pi i} \int_{\mathbb{R}} \sum c_{k}^{j} j !\left(x-z_{k}\right)^{-(j+1)}(g(x), h) d x\right|^{2} \\
& \leqslant \frac{1}{4 \pi} \int_{\mathbb{R}} \frac{|(g(x), h)|^{2}}{w^{2}(x)} d x \int_{\mathbb{R}} \frac{\left|\sum c_{k}^{j} j !\left(x-z_{k}\right)^{-(j+1)}\right|^{2}}{w^{2}(x)} d x \\
& \leqslant L \cdot\|h\|^{2} \int_{\mathbb{R}}\left|\sum c_{k}^{j} r_{k}^{j}(x)\right|^{2} w^{2}(x) d x
\end{aligned}
$$


Соединяя это с предыдущим, для любой линейной комбинации получаем двустороннюю оценку

$$
\left\|\sum_{k, j} c_{k}^{j} g_{k}^{j}\right\|_{\mathfrak{H}}^{2} \asymp \int_{\mathbb{R}}\left|\sum_{k, j} c_{k}^{j} r_{k}^{j}(x)\right|^{2} w^{2}(x) d x .
$$

Положим

$$
\mathfrak{N}:=\operatorname{clos} \operatorname{span}_{H_{-}^{2}\left(w^{2}\right)}\left\{r_{k}^{j}(z): 0 \leqslant j<m_{k}, z_{k} \in \sigma(A)\right\} .
$$

Напомним, что в силу теоремы 1.1 вес $w^{2}$ удовлетворяет условию $\left(\mathrm{A}_{2}\right)$. Таким образом, на основании теоремы 2.1 заключаем, что оператор $R$ непрерывно и непрерывно обратимо отображает $\mathfrak{H}$ на подпространство $\mathfrak{N}$ весового класса Харди $H_{-}^{2}\left(w^{2}\right)$. Обозначим через $\widetilde{A}$ оператор вида $(3.2)$, но в пространстве $\mathfrak{N}$, и докажем, что

$$
\widetilde{A}=R A R^{-1} .
$$

Для этого заметим, что если $p \in D_{A}$ записать в виде

$$
p=B h+(h, f) g,
$$

то $A p=h$. Простые вычисления с использованием (1.7) показывают, что

$$
\begin{aligned}
(R p)(z) & =(R B h)(z)+(h, f)(B g)(z) \\
& =(z \varphi(z))^{-1}\left\{\left(z B(I-z B)^{-1} h, f\right)+(h, f)\left((I-z B)^{-1} g, f\right)\right\} \\
& =(z \varphi(z))^{-1}\left\{-(h, f)+\left((I-z B)^{-1} h, f\right)+(h, f)(1-\varphi(z))\right\} \\
& =(z \varphi(z))^{-1}\left\{\left((I-z B)^{-1} h, f\right)-\varphi(z)(h, f)\right\} .
\end{aligned}
$$

Заметим еще, что функция $G(z):=(R h)(z)$, будучи элементом пространства $H_{-}^{2}\left(w^{2}\right)$, обладает свойством $\lim _{y \rightarrow-\infty} G(i y)=0$. Принимая во внимание перечисленные факты, приходим к равенству

$$
\begin{aligned}
(\widetilde{A} R p)(z) & =z(R p)(z)-\lim _{y \rightarrow-\infty} i y(R p)(i y) \\
& =\varphi^{-1}(z)\left((I-z B)^{-1} h, f\right)-(h, f)+(h, f)=(R h)(z)=(R A p)(z),
\end{aligned}
$$

т.е. $\widetilde{A} R=R A$.

Избавимся теперь от веса Макенхаупта $w^{2}$. Для этого рассмотрим оператор $S$ из $H_{-}^{2}\left(w^{2}\right)$ в $H_{-}^{2}$, определяемый формулой

$$
(S q)(z)=\mathbb{P}_{-} w_{+}(x+i 0) q(x), \quad q \in H_{-}^{2}\left(w^{2}\right),
$$

где через $q(x)$ обозначаются некасательные граничные значения $q \in H_{-}^{2}\left(w^{2}\right)$ на вещественной оси. При каждом $\lambda$ из области $\operatorname{Im} \lambda>0$ имеют место просто проверяемые равенства

$$
\begin{gathered}
\mathbb{P}_{-}\left(\frac{w_{+}(x+i 0)}{x-\lambda}\right)=\frac{w_{+}(\lambda)}{x-\lambda}, \\
\frac{1}{2(x-\lambda)}-\mathscr{K}_{w}\left(\frac{1}{x-\lambda}\right)=\frac{w_{+}^{-1}(\lambda) w_{+}(x+i 0)}{x-\lambda},
\end{gathered}
$$


где оператор $\mathscr{K}_{w}$ имеет вид

$$
\left(\mathscr{K}_{w} f\right)(x)=\frac{1}{2 \pi i} w_{+}(x+i 0) \int_{\mathbb{R}} w_{+}^{-1}(t+i 0)(t-x)^{-1} f(t) d t
$$

В силу теоремы 1.1 оператор $\mathscr{K}_{w}$ непрерывен в пространстве $L_{2}(\mathbb{R})$. Поэтому из (3.5) вытекает, что для любой конечной совокупности $\left\{\lambda_{k}\right\}$ из верхней полуплоскости

$$
\int_{\mathbb{R}} w^{2}(x)\left|\sum_{k} c_{k}\left(x-\lambda_{k}\right)^{-1}\right|^{2} d x \asymp \int_{\mathbb{R}}\left|\sum c_{k} w_{+}\left(\lambda_{k}\right)\left(x-\lambda_{k}\right)^{-1}\right|^{2} d x .
$$

Другими словами, $S$ - изоморфизм пространства $H_{-}^{2}\left(w^{2}\right)$ на $H_{-}^{2}$. Из (3.1) и (3.5) вытекает формула

$$
\left(S r_{k}^{j}\right)(z)=\left.\frac{d^{j}}{d \lambda^{j}} \frac{w_{+}(\lambda)}{z-\lambda}\right|_{\lambda=z_{k}}=R_{k}^{j}(z), \quad 0 \leqslant j<m_{k}, \quad \operatorname{Im} z<0 .
$$

Поэтому $S$ непрерывно и непрерьвно обратимо отображает $\mathfrak{N}$ на модельное подпространство $K_{B}$. Докажем теперь, что

$$
A_{m}=S \widetilde{A} S^{-1}
$$

Здесь сужение $S \mid \mathfrak{N}$ обозначается той же буквой $S$. В силу определений пространств $\mathfrak{N}, K_{B}$ равенство (3.7) достаточно доказать на каждом векторе $r_{k}^{j}$. Легко видеть, что

$$
\left(\widetilde{A} r_{k}^{0}\right)(z)=z_{k} r_{k}^{0}(z), \quad\left(\widetilde{A} r_{k}^{j}\right)(z)=j r_{k}^{j-1}(z)+z_{k} r_{k}^{j}(z), \quad 1 \leqslant j<m_{k}
$$

Поэтому из (3.6) вытекает

$$
\left(S \widetilde{A} r_{k}^{j}\right)(z)=j R_{k}^{j-1}(z)+z_{k} R_{k}^{j}(z), \quad 1 \leqslant j<m_{k}
$$

С другой стороны, имеем

$$
\begin{aligned}
\left(A_{m} S r_{k}^{(j)}\right)(z) & =\left(A_{m} R_{k}^{j}\right)(z)=\sum_{s=0}^{j} C_{j}^{s} w_{+}^{(j-s)}\left(z_{k}\right)\left(A_{m} r_{k}^{s}\right)(z) \\
& =z_{k} \sum_{s=0}^{j} C_{j}^{s} w_{+}^{(j-s)}\left(z_{k}\right) r_{k}^{s}(z)+\sum_{s=1}^{j} C_{j}^{s} s w_{+}^{(j-s)}\left(z_{k}\right) r_{k}^{(s-1)}(z) \\
& =z_{k} R_{k}^{j}(z)+\sum_{n=0}^{j-1} j C_{j-1}^{n} w_{+}^{(j-1-n)}\left(z_{k}\right) r_{k}^{n}(z)=z_{k} R_{k}^{j}(z)+j R_{k}^{j-1}(z)
\end{aligned}
$$

что вместе с (3.9) и доказывает (3.7). Поскольку $Q=S R$, то из (3.4), (3.7) следует подобие операторов $A$ и $A_{m}$ с оператором преобразования вида $(3.3)$. 
Достаточность. Поскольку

$$
U_{t}=Q V_{t} Q^{-1}, \quad V_{t}=\exp \left\{i A_{m} t\right\},
$$

и оператор $A_{m}$ диссипативен, то $U_{t}$ - полугруппа класса $C_{0}$. В работе [3] был вычислен спектр полугрупшы

$$
\sigma\left(V_{t}\right)=\left\{\lambda \in \mathbb{C}: \inf _{\operatorname{Im} z>0}\left(|B(z)|+\left|e^{i z t}-\lambda\right|\right)=0\right\},
$$

где $B$-произведение Бляшке с нулями на $\sigma(A)$ с учетом кратностей. Как и в [3], [5], из этой формулы выводится, что тип $V_{t}$, а значит, и $U_{t}$ вычисляется следующим образом:

$$
\omega=-\inf _{z_{k} \in \sigma(A)} \operatorname{Im} z_{k}
$$

Поэтому $U_{t} L_{2}$-устойчива.

Кроме формулы (3.10), которая является следствием теоремы о короне для алгебры $H^{\infty}$, в работе [3] была получена формула для полугрупшы $V_{t}$. Переходя к подобньм операторам, получим следующие результаты.

СлеДСТВИЕ. Если оператор $A$ генерирует $L_{2}$-устойчивую полугруппу $U_{t}=$ $\exp \{i A t\}, m o$

1) тип $U_{t}$ равен $\omega=-\inf _{z_{k} \in \sigma(A)} \operatorname{Im} z_{k}$ и имеет место формула

$$
U_{t} h=-\frac{1}{2 \pi i} \int_{\mathbb{R}} e^{i x t} g(x) \varphi^{-1}(x)\left((I-x B)^{-1} h, f\right) d x, \quad h \in \mathfrak{H} ;
$$

2) спектр $U_{t}$ вычисляется по формуле (3.10).

Впрочем, формулу (3.11) нетрудно проверить непосредственно.

Перейдем теперь к задаче подобия операторов $B \in \Lambda^{(\exp )}$ простейшему оператору интегрирования (0.5). Пусть $v^{2}$ - произвольный вес, удовлетворяющий условию $\left(\mathrm{A}_{2}\right)$ на $\mathbb{R}$. Тогда в области $\operatorname{Im} z<0$ имеет место интегральное представление [4]:

$$
v_{-}(z)=z \int_{\mathbb{R}_{+}} e^{i z t} g_{v}(t) d t
$$

с некоторой функцией $g_{v} \in L_{2}^{\operatorname{loc}}\left(\mathbb{R}_{+}\right)$. Здесь $v_{-}-$внешняя в области $\operatorname{Im} z<0$ функция, для которой $\left|v_{-}(x-i 0)\right|^{2} \stackrel{\text { п.в. }}{=} v^{2}(x), x \in \mathbb{R}$. Отметим, что при любом $a>0$ сужение $g_{v}$ на $[0, a]$ не входит в образ оператора $J_{a}$. Действительно, в противном случае после интегрирования по частям на $[0, a]$ мы получим в представлении (3.12) внешней функции $v_{-}$внутренний множитель $\exp \{i a z\}$.

Обозначим теперь через $A_{v}$ оператор вида $(0.1)$, где

$$
\mathfrak{H}=L_{2}(0, a), \quad B=J_{a}, \quad g(x)=g_{v}(x), \quad x \in[0, a],
$$

функция $f$ принадлежит $L_{2}(0, a)$ и такая, что пара $f, g$ согласована с $J_{a}$. В дальнейшем оператор $A_{v}$ будем называть оператором, порожденным весом $v^{2}$, а соответствуюшую вектор-функцию $g(z)$ обозначим через

$$
g_{v}(x, z):=\left(\left(I-z J_{a}\right)^{-1} g_{v}\right)(x) .
$$

Задача о генераторах $A_{v} L_{2}$-устойчивых полугрупп полностью решена. В нижеследующей формулировке $\varphi$, как и в общем случае, вычисляется по формуле (0.3). Предполагается также выполненным условие (0.4). 
Tеорема 3.2 [3], [5]. Onератор $A_{v}$ генерирует $L_{2}$-yстойчивую полугруппу $U_{t}=\exp \left\{i A_{v} t\right\}$ тогда и только тогда, когда $\sigma\left(A_{v}\right)$ лехсит в некоторой области $\operatorname{Im} z>\delta>0$ и вес $V^{2}(x):=|\varphi(x)|^{2} v^{-2}(x)$ удовлетворяет условию $\left(\mathrm{A}_{2}\right)$ на $\mathbb{R}$.

В безвесовой ситуации $\left(v^{2}(x) \equiv 1, x \in \mathbb{R}\right)$ эта теорема доказана в [3], а в общем случае - в [5].

Напомним, что вес $w^{2}$ определяется равенством (1.1), $g_{w}$ - формулой $(3.12)$, причем сужение $g_{w}$ на $[0, a]$ обозначается той же буквой, $W^{2}(x)=|\varphi(x)|^{2} w^{-2}(x)$. Через $а$ обозначается экспоненциальньй тип резольвенты $(I-z B)^{-1}, B \in \Lambda^{(\exp )}$.

Teоpema 3.3. Onератор $A$ генерирует $L_{2}$-устойчивую полугруппу $U_{t}=$ $\exp \{i A t\}$ в том и только том случае, когда выполнена совокупность требований:

1) $\sigma(A)$ лежит в некоторой полуплоскости $\operatorname{Im} z>\delta>0$;

2) веса $w^{2}, W^{2}$ удовлетворяют условию $\left(\mathrm{A}_{2}\right)$ на $\mathbb{R}$;

3) оператор $B$ подобен $J_{a}$, m.е. $B=S J_{a} S^{-1}$, причем $S g_{w}=g$.

ДоказАТЕЛЬство. Необходимость. Условия 1), 2) выполняются (§1). Далее, при доказательстве теоремы 3.1 была получена двусторонняя оценка для линейных комбинаций корневых векторов операторов $A$ и $\widetilde{A}$ :

$$
\left\|\sum_{j, k} c_{k}^{j} g_{k}^{j}\right\|_{\mathfrak{H}}^{2} \asymp \int_{\mathbb{R}}\left|\sum_{j, k} c_{k}^{j} r_{k}^{j}(x)\right|^{2} w^{2}(x) d x .
$$

Построим по весу Макенхаупта $w^{2}$ оператор $A_{w}$ в пространстве $L_{2}(0, a)$ с тем же спектром, что и у оператора $A$. Для этого заметим, что из (0.3) и леммы 1.2 вытекает, что целая функция экспоненциального типа $\Phi(z):=z^{-1}(1-\varphi(z))$ удовлетворяет условию

$$
\int_{\mathbb{R}}|\Phi(x)|^{2} w^{-2}(x) d x<\infty .
$$

Из условия $(0.4)$ получаем неравенства $h_{\Phi}(\pi / 2) \leqslant 0, h_{\Phi}(-\pi / 2) \leqslant a$. В работе [4] доказан весовой аналог теоремы Винера-Пэли, согласно которому $\Phi$ допускает интегральное представление

$$
\Phi(z)=\left(g_{w}(t, z), f\right)_{L_{2}(0, a)}
$$

с ядром $g_{w}(t, z)$ вида (3.13). Покажем, что пара $f, g_{w}$ согласована с оператором $J_{a}$. В самом деле, если $f=J_{a}^{*} v,\left(v, g_{w}\right)=-1$, то

$$
\begin{aligned}
\varphi(z) & =1-z \Phi(z)=1-z\left(J_{a}\left(I-z J_{a}\right)^{-1} g_{w}, v\right) \\
& =1-\left(\left(I-z J_{a}\right)^{-1} g_{w}, v\right)+\left(g_{w}, v\right)=-\left(\left(I-z J_{a}\right)^{-1} g_{w}, v\right)
\end{aligned}
$$

В [4], [5] доказано, что

$$
m\|h\|^{2} \leqslant \int_{\mathbb{R}} w^{-2}(x)\left|\left(\left(I-x J_{a}\right)^{-1} g_{w}, h\right)\right|^{2} d x \leqslant M\|h\|^{2}, \quad h \in L_{2}(0, a) .
$$


Вспоминая теперь выражение для веса $W^{2}$, приходим к оценке

$$
\int_{\mathbb{R}} W^{2}(x) d x=\int_{\mathbb{R}}|\varphi(x)|^{2} w^{-2}(x) d x \leqslant M\|v\|^{2},
$$

что невозможно, так как $W^{2}$ удовлетворяет условию $\left(\mathrm{A}_{2}\right)$.

Итак, оператор $A_{w}$, построенный по $J_{a}$ и паре функций $f, g_{w} \in L_{2}(0, a)$ ( $f$ взята из представления (3.15)), имеет тот же спектр, что и $A$. Поскольку вьполнены все условия теоремы 3.2 , то полугруппа $V_{t}=\exp \left\{i A_{w} t\right\} L_{2}$-устойчива и, стало быть, для нее справедлив аналог оценки (3.14):

$$
\left\|\sum_{j, k} c_{k}^{j} g_{w}^{(j)}\left(t, z_{k}\right)\right\|_{L_{2}(0, a)}^{2} \asymp \int_{\mathbb{R}}\left|\sum_{j, k} c_{k}^{j} r_{k}^{j}(x)\right|^{2} w^{2}(x) d x .
$$

Таким образом, оператор $S$, определенный на линейных комбинациях корневых векторов равенствами

$$
S\left(\sum_{j, k} c_{k}^{j} g_{w}^{(j)}\left(t, z_{k}\right)\right)=\sum_{j, k} c_{k}^{j} g_{k}^{j}
$$

в силу теоремы 2.1 продолжается замыканием до изоморфизма пространств $L_{2}(0, a)$ и $\mathfrak{H}$. Заметим, что эта теорема применима к оператору $A_{w}$, поскольку тип резольвенты $\left(I-z J_{a}\right)^{-1}$ равен $a$. С другой стороны, из леммы 1.2 и весового аналога теоремы Винера-Пэли [4] следует сушествование линейного оператора $S_{1}: \mathfrak{H} \rightarrow$ $L_{2}(0, a)$, для которого

$$
(g(z), h)_{\mathfrak{H}}=\left(g_{w}(t, z), S_{1} h\right)_{L_{2}(0, a)}, \quad h \in \mathfrak{H}, \quad z \in \mathbb{C} .
$$

Из левого неравенства (3.16) выводим, что оператор $S_{1}$ ограничен. Сопоставление (3.17) и равенства

$$
S_{1}^{*}\left(I-z J_{a}\right)^{-1} g_{w}=(I-z B)^{-1} g, \quad z \in \mathbb{C},
$$

показывают, что $S=S_{1}^{*}$. Таким образом, $B=S J_{a} S^{-1}, S g_{w}=g$.

Достаточность. Из условия 3$)$ следует, что $A$ подобен некоторому оператоpy $A_{w}$, который в силу теоремы 3.2 генерирует $L_{2}$-устойчивую полугруппу. Поэтому полугруппа $U_{t}=\exp \{i A t\}$ обладает этим же свойством.

ЗАмЕчАнИЕ. Как и в теореме 3.1 , здесь тоже можно явно записать вид оператора преобразования $S$. Таким оператором будет

$$
S f=\frac{1}{\sqrt{2 \pi i}} \int_{\mathbb{R}} g(x) w_{-}^{-1}(x-i 0)\left(f, e^{i x t}\right)_{L_{2}(0, a)} d x, \quad f \in L_{2}(0, a) .
$$

Ограниченность $S$ обеспечивается оценкой (1.14), а его непрерывная обратимость вытекает из условия теоремы 3.3. Формулу (3.18) мы приводим здесь без доказательства, поскольку в дальнейшем она не понадобится. Пример, иллюстрирующий теорему 3.3 , будет приведен в $\S 4$.

Полученные результаты пригодны для решения обшей задачи об описании генератором вида $(0.1)$ полугрупп класса $C_{0}$. Переход к $L_{2}$-устойчивым полугруппам осуществляется с помощью сдвига спектра $\left\{z_{k}\right\}_{-\infty}^{+\infty}$ вдоль мнимой оси. Не вдаваясь в подробности, сформулируем, например, соответствуюший аналог теоремы 3.1. 
Tеорема 3.4. Onератор A генерирует полугруппу $U_{t}=\exp \{i A t\} \kappa л а с с а C_{0}$ тогда и только тогда, когда

1) $\inf _{z_{k} \in \sigma(A)} \operatorname{Im} z_{k}>\sigma>-\infty$;

2) оператор $A-i \sigma I$ подобен модельному оператору (3.2) со спектром $\left\{z_{k}-i \sigma\right\}_{-\infty}^{+\infty}$

\section{§4. Безусловные базисы, составленные из значений квазиэкспонент}

Начнем с примеров квазиэкспонент. Ими будут вектор-функции вида

$$
e(z):=(I-z B)^{-1} g, \quad B \in \Lambda^{(\exp )}, \quad g \in \mathfrak{H}
$$

которые нам уже встречались раньше. В самом деле, так как $\tilde{e}(z)=B(I-z B)^{-1} g$, TO

$$
\begin{gathered}
(\tilde{e}(\lambda), e(z))=\left(B(I-\lambda B)^{-1} g, e(z)\right)=\left(e(\lambda), B^{*} e(z)\right) \\
\operatorname{Im}\left(\sum c_{k} \tilde{e}\left(\lambda_{k}\right), \sum c_{k} e\left(\lambda_{k}\right)\right)=(\operatorname{Im} B h, h) \geqslant 0, \quad h=\sum c_{k} e\left(\lambda_{k}\right)
\end{gathered}
$$

Выбирая должным образом $\mathfrak{H}, B, g$, можно получить конкретные интересные примеры квазиэкспонент. В этой работе важную роль будет играть один из них:

$$
e(z)=\frac{d}{d t} \int_{0}^{t} g(t-s) e^{i z s} d s, \quad g \in L_{2}(0, a)
$$

что отвечает выбору

$$
\mathfrak{H}=L_{2}(0, a), \quad B=J_{a}, \quad g=g(t) .
$$

В частности, экспонента $\exp \{i z t\}$ является квазиэкспонентой со значениями в каждом пространстве $L_{2}(0, a)$.

Поскольку для нас представляют интерес только регулярные квазиэкспоненты, то примерами (4.1) можно ограничиться, так как справедлива

ТЕОРема 4.1. Кажсдая регулярная квазиэкспонента е: $\mathbb{C} \rightarrow \mathfrak{H}$ допускает единственное представление вида (4.1), в котором $B$ - одноклеточный оператор класса $\Lambda^{(\mathrm{exp})}$. При этом әкспоненциальные типь е и резольвенты $(I-z B)^{-1}$ равньь.

ДокАЗАТЕЛьство. Сушествует хотя бы один безусловный базис пространства $\mathfrak{H}$ вида

$$
\left\{e\left(\lambda_{k}\right): \lambda_{k} \in \Lambda\right\}, \quad \inf _{k} \lambda_{k}>0 \text {. }
$$

Стандартные рассуждения с выбрасыванием одного элемента (например, $e\left(\lambda_{1}\right)$ ) показывают, что последовательность $\Lambda$ содержится в множестве корней целой функции

$$
\varphi(z):=\left(z-\lambda_{1}\right)(e(z), h)
$$


экспоненциального типа и, стало быть, сходится ряд (см. [11]):

$$
\sum_{\lambda_{k} \in \Lambda}\left|\lambda_{k}\right|^{-2}<\infty
$$

На элементах базиса (4.3) зададим оператор $B$ формулой

$$
B e\left(\lambda_{k}\right)=\lambda_{k}^{-1}\left(e\left(\lambda_{k}\right)-e(0)\right), \quad \lambda_{k} \in \Lambda,
$$

и докажем, что он ограничен в пространстве $\mathfrak{H}$. Для любой линейной комбинации $h=\sum c_{k} e\left(\lambda_{k}\right)$ имеем

$$
\begin{aligned}
\|B h\|^{2} & \leqslant 2\left\|\sum c_{k} \lambda_{k}^{-1} e\left(\lambda_{k}\right)\right\|^{2}+2\|e(0)\|^{2}\left|\sum c_{k}\left\|e\left(\lambda_{k}\right)\right\|\left(\lambda_{k}\left\|e\left(\lambda_{k}\right)\right\|\right)^{-1}\right|^{2} \\
& \leqslant M \sum\left|c_{k}\right|^{2}\left\|e\left(\lambda_{k}\right)\right\|^{2}+2\|e(0)\|^{2} \sum\left|\lambda_{k}\right|^{-2}\left\|e\left(\lambda_{k}\right)\right\|^{-2} \sum\left|c_{k}\right|^{2}\left\|e\left(\lambda_{k}\right)\right\|^{2} .
\end{aligned}
$$

Из условия 1) определения 1 при $z=0$ получаем равенства

$$
\frac{\left(e\left(\lambda_{k}\right), e(0)\right)-\|e(0)\|^{2}}{\lambda_{k}\left\|e\left(\lambda_{k}\right)\right\|}=\left(\frac{e\left(\lambda_{k}\right)}{\left\|e\left(\lambda_{k}\right)\right\|}, h_{0}\right), \quad \lambda_{k} \in \Lambda, \quad h_{0} \in \mathfrak{H},
$$

из которых вытекает сходимость ряда

$$
\sum_{\lambda_{k}}\left|\lambda_{k}\right|^{-2}\left\|e\left(\lambda_{k}\right)\right\|^{-2}<\infty
$$

поскольку $e(0) \neq 0$. Действительно, если предположить, что $e(0)=0$, то из условия 2) определения 1 следует противоречие:

$$
\operatorname{Im}\left\{\frac{1}{\lambda_{k}}\left|c_{k}\right|^{2}\left\|e\left(\lambda_{k}\right)\right\|^{2}\right\} \geqslant 0, \quad \operatorname{Im} \lambda_{k}>0
$$

Поэтому из (4.6) выводим оценку

$$
\|B h\|^{2} \leqslant K \sum\left|c_{k}\right|^{2}\left\|e\left(\lambda_{k}\right)\right\|^{2} \leqslant K_{1}\|h\|^{2}
$$

для всюду плотного в $\mathfrak{H}$ множества векторов.

Убедимся теперь, что $\sigma(B)=\{0\}$. Для этого докажем, что $B$ является оператором Гильберта-Шмидта без ненулевых собственных чисел. Поскольку нормированная система $\left(\left\|e\left(\lambda_{k}\right)\right\|\right)^{-1} e\left(\lambda_{k}\right)$ образует базис Рисса пространства $\mathfrak{H}$, то с учетом (4.5) получаем

$$
\begin{aligned}
\sum_{\lambda_{k} \in \Lambda}\left\|B e\left(\lambda_{k}\right)\right\|^{2}\left\|e\left(\lambda_{k}\right)\right\|^{-2} & =\sum_{\lambda_{k} \in \Lambda}\left\|\lambda_{k}^{-1}\left(e\left(\lambda_{k}\right)-e(0)\right)\right\|^{2}\left\|e\left(\lambda_{k}\right)\right\|^{-2} \\
& \leqslant C\left(\sum\left|\lambda_{k}\right|^{-2}+\sum\left|\lambda_{k}\right|^{-2}\left\|e\left(\lambda_{k}\right)\right\|^{-2}\right)<\infty
\end{aligned}
$$


т.е. $B \in \mathfrak{S}_{2}$. Докажем теперь, что уравнение

$$
(I-\mu B) h=0, \quad \mu \neq 0,
$$

имеет только лишь тривиальное решение. Для этого сначала заметим, что

$$
B e(\lambda)=\lambda^{-1}(e(\lambda)-e(0)) \quad \forall \lambda \in \mathbb{C} .
$$

Действительно, снова из условия 1) определения 1 вытекает, что для каждого вектора $g=\sum c_{k} e\left(z_{k}\right)$ сушествует такой вектор $h_{g} \in \mathfrak{H}$, что

$$
(\tilde{e}(\lambda), g)=\left(e(\lambda), h_{g}\right)
$$

для всех $\lambda \in \mathbb{C}$. Если это равенство рассмотреть только на последовательности $\Lambda$, то из определения $B$ следует, что

$$
\left(\tilde{e}\left(\lambda_{k}\right), g\right)=\left(e\left(\lambda_{k}\right), B^{*} g\right), \quad \lambda_{k} \in \Lambda
$$

Таким образом, из двух последних равенств вытекает, что $B^{*} g=h_{g}$ и, стало быть, (4.7) имеет место. Вернемся теперь к нашему уравнению, предположив сначала, что $\mu \notin \Lambda$. Рассмотрим вектор-функцию

$$
h_{k}=\frac{\mu e(\mu)-\lambda_{k} e\left(\lambda_{k}\right)}{\mu-\lambda_{k}}, \quad \lambda_{k} \in \Lambda
$$

и, воспользовавшись формулой (4.7), вычислим

$$
(I-\mu B) h_{k}=h_{k}-\mu \frac{\mu B e(\mu)-\lambda_{k} B\left(\lambda_{k}\right)}{\mu-\lambda_{k}}=e\left(\lambda_{k}\right) .
$$

Если $\mu=\lambda_{k} \in \Lambda$, то положим

$$
h_{k}=e\left(\lambda_{k}\right)+\lambda_{k} e^{\prime}\left(\lambda_{k}\right), \quad \lambda_{k} \in \Lambda
$$

Дифференцируя (4.7), найдем

$$
\lambda^{2} B e^{\prime}(\lambda)=\lambda e^{\prime}(\lambda)-e(\lambda)+e(0)
$$

Поэтому снова

$$
\left(I-\lambda_{k} B\right) h_{k}=h_{k}-\lambda_{k}\left(B e\left(\lambda_{k}\right)+\lambda_{k} B e^{\prime}\left(\lambda_{k}\right)\right)=e\left(\lambda_{k}\right)
$$

Следовательно, в обоих случаях в образе оператора $I-\mu B$ содержится безусловный базис пространства $\mathfrak{H}$, т.е. $(I-\mu B) \mathfrak{H}=\mathfrak{H}$. Поэтому $\sigma(B)=\{0\}$. Далее, условие 2) из определения квазиэкспоненты означает, что оператор $B$ диссипативен:

$$
\operatorname{Im}\left(\sum c_{k} \tilde{e}\left(\lambda_{k}\right), \sum c_{k} e\left(\lambda_{k}\right)\right)=(\operatorname{Im} B h, h) \geqslant 0, \quad h=\sum c_{k} e\left(\lambda_{k}\right)
$$

Из (4.7) вытекает представление $e(z)=(I-z B)^{-1} e(0)$, в котором резольвента $(I-z B)^{-1}$ имеет конечный экспоненциальньй тип. В самом деле, для всюду 
плотного в $\mathfrak{H}$ множества конечных сумм $f=\sum_{k \geqslant 0} c_{k} B^{k} e(0)$ экспоненциальные типы функций

$$
\left(B(I-z B)^{-1} f, g\right)=\sum_{k \geqslant 0} c_{k}\left(e(z),\left(B^{*}\right)^{k+1} g\right), \quad g \in \mathfrak{H}
$$

ограничены числом $a$, где $a$ - тип квазиэкспоненты $e$. Для сжимаюшей полугрупшы $V_{t}:=\exp \left\{-i B^{-1} t\right\}$ имеем [2]:

$$
\int_{0}^{\infty} e^{i z t}\left(V_{t} f, g\right) d t=-i\left(B(I-z B)^{-1} f, g\right), \quad \operatorname{Im} z>0 .
$$

Из теоремы Винера-Пэли вытекает, что $U_{t} f=0$ при $t>a$ сначала для конечных сумм, а потом и для всех $f \in \mathfrak{H}$. Теперь из (4.8) следует, что тип $(I-z B)^{-1}$ не больше $a$ и, стало быть, он равен $a$. Таким образом, $B \in \Lambda^{(\exp )}$.

Поскольку е регулярна, то $g:=e(0)$ является циклическим вектором оператора $B$. Поэтому $B$ одноклеточен и имеет ядерную мнимую компоненту [1]. Впрочем, ядерность $\operatorname{Im} B$ легко получить и без ссылки на этот довольно тонкий результат теории одноклеточных операторов:

$$
\begin{aligned}
& \sum_{\lambda_{k} \in \Lambda}\left|\frac{\left(\left(B-B^{*}\right) e\left(\lambda_{k}\right), e\left(\lambda_{k}\right)\right)}{\left\|e\left(\lambda_{k}\right)\right\|^{2}}\right| \leqslant 2 \sum_{\lambda_{k} \in \Lambda} \frac{\left|\left(e\left(\lambda_{k}\right), e(0)\right)\right|}{\left|\lambda_{k}\right|\left\|e\left(\lambda_{k}\right)\right\|^{2}} \\
& \quad \leqslant 2\left\{\sum_{\lambda_{k} \in \Lambda}\left|\left(e\left(\lambda_{k}\right), e(0)\right)\right|^{2}\left\|e\left(\lambda_{k}\right)\right\|^{-2}\right\}^{1 / 2}\left\{\sum_{\lambda_{k} \in \Lambda}\left|\lambda_{k}\right|^{-2}\left\|e\left(\lambda_{k}\right)\right\|^{-2}\right\}^{1 / 2}<\infty .
\end{aligned}
$$

Единственность представления (4.1) очевидна.

Оператор $B$ в представлении (4.2) одноклеточен и имеет одномерную мнимую часть, однако не все эти квазиэкспоненты регулярны. Ниже будет дано описание всех регулярных квазиэкспонент вида (4.2).

Введем один важный подкласс квазиэкспонент (4.2), которые являются регулярньми. Они каноническим образом строятся по $\mathrm{A}_{2}$-весам Макенхаупта на вещественной прямой. К определениям 1, 2 из введения добавим следующее

ОПреДЕЛЕНИЕ 3 . Пусть $w^{2}$ - произвольный вес Макенхаупта на $\mathbb{R}$. Квазиэкспоненту

$$
e_{w}(z):=\frac{d}{d t} \int_{0}^{t} g_{w}(t-s) e^{i z s} d s
$$

со значениями в пространстве $L_{2}(0, a)$, где $g_{w}$ принадлежит $L_{2}^{\text {loc }}\left(\mathbb{R}_{+}\right)$и отвечает весу $w^{2}$ в силу формулы (3.12), будем называть $w$-квазиэкспонентой.

Квазиэкспоненты этого класса были введены в [4] (там они назывались ядрами, порожденньми весами Макенхаупта) и достаточно подробно изучались в [4], [5]. Сформулируем основной результат о базисах, составленных из функций вида (4.9), в которых последовательность $\Lambda$ может иметь кратные элементы. Через $m_{k}$ обозначается кратность элемента $\lambda_{k} \in \Lambda$. Предполагаются также выполненными условия

$$
\inf _{\lambda_{k} \in \Lambda} \operatorname{Im} \lambda_{k}>b>-\infty, \quad \sup _{\lambda_{k} \in \Lambda} m_{k}<\infty .
$$

Для определенности будем считать, что $b \leqslant 0$. 
ТЕОРема 4.2 [4], [5]. Пусть последовательность $\Lambda$ удовлетворяет условиям (4.10). Тогда семейство функиий

$$
\left\{e_{w}^{(j)}\left(\lambda_{k}\right): \lambda_{k} \in \Lambda, 0 \leqslant j<m_{k}\right\}
$$

образует безусловный базис пространства $L_{2}(0, a)$ тогда и только тогда, когда $\Lambda$ совпадает с множеством корней целой функции $\varphi$ әкспоненциального типа, которая удовлетворяет условиям:

1) $h_{\varphi}(\pi / 2)=0, h_{\varphi}(-\pi / 2)=a$;

2) вес $W^{2}(x):=\left|w_{-}(x+b i)\right|^{-2}|\varphi(x+b i)|^{2}$ удовлетворяет условию $\left(\mathrm{A}_{2}\right)$ на вещественной прямой;

3) последовательность $\mu_{k}=\lambda_{k}-b i\left(\lambda_{k} \in \Lambda\right)$ отделима в смисле Карлесона:

$$
\inf _{k} \prod_{j \neq k}\left|\frac{\mu_{k}-\mu_{j}}{\mu_{k}-\bar{\mu}_{j}}\right|>0
$$

ЗАмЕчАнИЕ. Формулировку этой теоремы можно уточнить следующим образом. В случае базисности системы (4.11) условия 2), 3) вьполняются при любом $b \leqslant 0$, удовлетворяющем первому неравенству (4.10). Если же условия теоремы выполнены при каком-нибудь таком $b$, то семейство (4.11) образует безусловный базис пространства $L_{2}(0, a)$.

СлеДСтвИЕ. Для любого веса Макенхаупта $w^{2}$ на $\mathbb{R}$ w-квазиэкспонента $e_{w}$ регулярна.

В самом деле, семейства $\left\{e_{w}\left(\lambda_{k}\right)\right\}_{-\infty}^{+\infty}$, где $\lambda_{k}=\frac{2 \pi k}{a}+i \beta(k \in \mathbb{Z}, \beta \in \mathbb{R})$, образуют безусловные базисы $L_{2}(0, a)$ для любого $\mathrm{A}_{2}$-веса $w^{2}$, поскольку функция $\varphi(z):=e^{i z a}-e^{\beta a}$ удовлетворяет условиям теоремы 4.2 .

ОПРЕДЕЛЕНИЕ 4. Две квазиэкспоненты $e_{1}, e_{2}$ со значениями в пространствах $\mathfrak{H}_{1}, \mathfrak{H}_{2}$ называются изоморфными, если сушествует изоморфизм ${ }^{1} S$ пространства $\mathfrak{H}_{1}$ на $\mathfrak{H}_{2}$ такой, что $S e_{1}(\lambda)=e_{2}(\lambda)$ для всех $\lambda \in \mathbb{C}$.

Ясно, что изоморфные квазиэкспоненты могут быть регулярньми лишь только одновременно. Таким образом, регулярными будут $w$-квазиэкспоненты для любого веса Макенхаупта $w^{2}$ и все их изоморфные образы. Основной результат этого параграфа состоит в том, что других регулярных квазиэкспонент нет. После уже проделанной работы этот факт является несложньм следствием теоремы 3.3 .

ТЕОРема 4.3. Пусть е - квазиэкспонента со значениями в пространстве $\mathfrak{H}$, әкспоненциальный тип которого равен $a<\infty$. Тогда регулярность $е$ равносильна выполнению двух условий:

1) вес $w^{2}(x):=\|е(x)\|^{2}$ удовлетворяет условию $\left(\mathrm{A}_{2}\right)$ на $\mathbb{R} ;$

2) квазиэкспонента е изоморфна е

\footnotetext{
${ }^{1}$ Изоморфизмом назьвается непрерывньй и непрерьвно обратимый линейный оператор $S$ из $\mathfrak{H}_{1}$ на $\mathfrak{H}_{2}$.
} 
ДокАЗАТЕЛЬство. Пусть $e-$ регулярная квазиэкспонента и семейство

$$
\left\{e\left(\lambda_{k}\right): \lambda_{k} \in \Lambda\right\}, \quad \operatorname{Im} \lambda_{k} \geqslant \delta>0
$$

образует безусловньй базис пространства $\mathfrak{H}$. Тогда $\Lambda$ содержится в множестве корней функции $\varphi$ вида (4.4), которую с учетом теоремы 4.1 теперь можно записать в виде

$$
\varphi(z)=\left(z-\lambda_{1}\right)\left((I-z B)^{-1} g, h\right)
$$

где $g \in \mathfrak{H}, B$ - одноклеточный оператор класса $\Lambda^{(\exp )}$. Легко видеть, что

$$
\varphi(z)-\varphi(0)=-z\left((I-z B)^{-1} g, f\right), \quad f:=-h+\bar{\lambda}_{1} B^{*} h
$$

Без ограничения обшности полагаем $\varphi(0)=1$, и следовательно, функция $\varphi$ имеет вид (0.3). Рассматривая оператор $K h:=B h+(h, f) g$, легко установить, что во-первых, $\Lambda$ в точности совпадает с множеством корней $\varphi$ и, во-вторых, пара $f$, $g$ согласована с оператором $B$. Ясно, что $\operatorname{Ker} B=\operatorname{Ker} B^{*}=\{0\}$, и в силу теоремы 4.1 тип $(I-z B)^{-1}$ равен $a$. Для оператора $A$, определенного формулой $(0.1)$, имеем

$$
A e\left(\lambda_{k}\right)=\lambda_{k} e\left(\lambda_{k}\right), \quad \lambda_{k} \in \Lambda, \quad \operatorname{Im} \lambda_{k} \geqslant \delta>0
$$

Поэтому полугруппа $U_{t}=\exp \{i A t\} \quad L_{2}$-устойчива. На основании теоремы 2.1 заключаем, что выполняются условия (0.4). Другим словами, мы находимся в условиях применимости теоремы 3.3 , согласно которой вес

$$
w^{2}(x)=\|g(x)\|^{2}=\|e(x)\|^{2}, \quad x \in \mathbb{R},
$$

удовлетворяет условию $\left(\mathrm{A}_{2}\right)$ и $B=S J_{a} S^{-1}, S g_{w}=g$. Следовательно,

$$
e(z)=(I-z B)^{-1} g=S\left(I-z J_{a}\right)^{-1} g_{w}=S e_{w}(z)
$$

где $S$ - изоморфизм пространств $L_{2}(0, a)$ и $\mathfrak{H}$, т.е. е и $e_{w}$ изоморфны.

Обратное утверждение очевидно. Надо только учесть, что экспоненциальньй тип $e_{w}$ в $L_{2}(0, a)$ равен $a$.

ЗАмЕчАнИЕ 1. Утверждение теоремы остается в силе, если вместо регулярности $е$ потребовать формально меньше: сушествует такая последовательность $\Lambda=\left\{\lambda_{k}\right\}_{-\infty}^{+\infty}\left(\operatorname{Im} \lambda_{k} \geqslant \delta>0\right)$ с ограниченными кратностями $m_{k}$, что семейство $\left\{e^{(s)}\left(\lambda_{k}\right):=\lambda_{k} \in \Lambda, 0 \leqslant s<m_{k}\right\}$ образует безусловный базис $\mathfrak{H}$.

ЗАмечАниЕ 2. Теорема 4.3 дает положительный ответ на оба вопроса заметки [7], которые (в наших терминах) формулировались так: пусть квазиэкспонента $(I-z B)^{-1} g\left(B \in \Lambda^{(\exp )}\right)$ регулярна. Верно ли, что выполняются условия 1$\left.), 2\right) ?$ Теорема 4.3 впервые была анонсирована в работе [8], в которой было введено понятие квазиэкспоненты и сформулированы почти все результаты этого параграфа. Недавно А.М. Минкин [16], не зная о работе [8], применяя другие соображения, снова доказал необходимость условия 1$)$ для регулярности $(I-z B)^{-1} g$ при дополнительном предположении $\operatorname{dim}\left(B-B^{*}\right) \mathfrak{H}<\infty$.

Из теорем 4.2, 4.3 вытекает следующий результат. 
ТЕОРемА 4.4. Пусть е - регулярная квазиэкспонента әкспоненииального типа а со значениями в пространстве $\mathfrak{H}$, последовательность $\Lambda$ удовлетворяет условиям (4.10). Тогда семейство векторов

$$
\left\{e^{(j)}\left(\lambda_{k}\right): 0 \leqslant j<m_{k}, \lambda_{k} \in \Lambda\right\}
$$

образует безусловный базис пространства $\mathfrak{H}$ тогда и только тогда, когда $\Lambda$ совпадает с множеством корней целой функиии $\varphi$, которая удовлетворяет условиям 1)-3) теоремы 4.2 с $w^{2}(x)=\|e(x)\|^{2}(x \in \mathbb{R})$. При этом кажсдий базис (4.12) изоморфен базису пространства $L_{2}(0, a)$ вида (4.11), построенному nо весу $w^{2}$.

В качестве приложения изложенных результатов дадим ответ на следующий вопрос: для каких $g \in L_{2}(0, a)$ квазиэкспонента (4.2) регулярна? Для этой цели рассмотрим множество всех функций $v$, аналитических в области $\operatorname{Im} z<0$ и представимых там в виде

$$
v(z)=s(z) w(z), \quad \operatorname{Im} z<0,
$$

где $w$ - такие внешние функции, что веса $|w(x-i 0)|^{2}$ удовлетворяют условию $\left(\mathrm{A}_{2}\right)$ на $\mathbb{R}$, а $s$ - внутренние функции, для которых

$$
\inf _{\operatorname{Im} z<0}\left\{|s(z)|+\left|e^{-i z a}\right|\right\}>0
$$

Легко видеть, что каждая функция $v$ допускает представление

$$
v(z)=z \int_{0}^{\infty} e^{-i z t} g(t) d t, \quad g \in L_{2}^{\text {loc }}\left(\mathbb{R}_{+}\right) .
$$

Представляя каждую функцию (4.13) в виде (4.14), получим множество функций $g \in L_{2}^{\text {loc }}\left(\mathbb{R}_{+}\right)$, которое будем обозначать через $\mathscr{M}$. Множество сужений $g \in \mathscr{M}$ на сегмент $[0, a]$ обозначим через $\mathscr{M}_{a}$. Отметим, что в том случае, когда в $(4.13)$ множитель $s$ отсутствует, функции $g \in \mathscr{M}_{a}$ нам встречались при определении $w$-квазиэкспоненты, построенной по весу $|w(x-i 0)|^{2}$.

Комбинируя теорему Сарасона о коммутанте [15] с теоремой о короне [17], приходим к следующему результату.

ТЕОрема 4.5. Квазиэкспонента (4.2) регулярна тогда и только тогда, когда $g \in \mathscr{M}_{a}$.

ДокАЗАТЕЛЬСтво. Из теоремы 4.3 вытекает, что в случае регулярности $e(z)=\left(I-z J_{a}\right)^{-1} g$ сушествует изоморфизм $S$ пространства $L_{2}(0, a)$ такой, что $S\left(I-z J_{a}\right)^{-1} g_{w}=(I-z J)^{-1} g$, где $g_{w}$ отвечает некоторому $\mathrm{A}_{2}$-весу $w^{2}$ в силу формулы (3.12). Поэтому

$$
S J_{a}=J_{a} S, \quad S g_{w}=g
$$

т.е. $S$ принадлежит коммутанту оператора $J_{a}$. Теорема Сарасона [15] утверждает, что $S$ унитарно эквивалентен оператору

$$
(\widetilde{S} f)(z)=\mathbb{P}_{a} \tilde{s}(z) f(z), \quad \tilde{s} \in H_{+}^{\infty}, \quad f \in K_{a}:=H_{+}^{2} \ominus e^{i z a} H_{+}^{2},
$$


где $\mathbb{P}_{a}$ - ортопроектор из $H_{+}^{2}$ на $K_{a}$. Далее, $\widetilde{S}$ является изоморфизмом на $K_{a}$ тогда и только тогда, когда существует $p \in H_{+}^{\infty}$ такая, что

$$
(1-\tilde{s} p) u \in e^{i z a} H_{+}^{2}
$$

для всех $u \in H_{2}^{+}[15]$. Взяв в качестве $u(z)=(z+i)^{-1}$, получим

$$
1-\tilde{s}(z) p(z)=e^{i z a} v(z)(z+i), \quad v \in H_{+}^{2} .
$$

Поэтому $\psi(z):=v(z)(z+i) \in H_{+}^{\infty}$. Таким образом, если оператор $\widetilde{S}$ непрерывно обратим, то найдутся функции $p, \psi \in H_{+}^{\infty}$ такие, что

$$
\tilde{s}(z) p(z)+e^{i a z} \psi(z) \equiv 1, \quad \operatorname{Im} z>0 .
$$

Функция $\tilde{s}$ по оператору $\widetilde{S}$ определяется неоднозначно. Покажем, что ее можно выбрать так, чтобы $|\tilde{s}(x+i 0)| \asymp 1$ почти всюду на $\mathbb{R}$. В самом деле, $\tilde{s}_{0}(z):=\tilde{s}(z)+m e^{i z a}$ при подходящем выборе константы $m$ удовлетворяет этому условию. Вместе с тем $\tilde{s}_{0}$ наследует свойства $(4.16),(4.17)$ функции $\tilde{s}$ :

$(\widetilde{S} f)(z)=\mathbb{P}_{a} \tilde{s}(z) f(z)=\mathbb{P}_{a} \tilde{s}_{0}(z) f(z), \quad\left(1-\tilde{s}_{0} p\right) u=(1-\tilde{s} p) u-m e^{i z a} p u \in e^{i z a} H_{+}^{2}$.

Теперь с помощью преобразования Фурье-Лапласа снова вернемся в пространство $L_{2}(0, a)$. Для этого представим функцию $\tilde{s}$ в виде

$$
\tilde{s}(z)=-i z \int_{0}^{\infty} e^{i z t} k(t) d t, \quad k \in L_{2}^{\mathrm{loc}}\left(\mathbb{R}_{+}\right), \quad \operatorname{Im} z>0
$$

и введем в $L_{2}(0, a)$ оператор

$$
(S h)(t)=\frac{d}{d t} \int_{0}^{t} k(t-s) h(s) d s, \quad h \in L_{2}(0, a), \quad 0 \leqslant t \leqslant a .
$$

Если $t \in \mathbb{R}_{+}$и $\operatorname{Im} z>0$, то

$$
\int_{0}^{\infty} e^{i z t} \frac{d}{d t} \int_{0}^{t} k(t-s) h(s) d s d t=\tilde{s}(z) \hat{h}(z), \quad \hat{h}(z):=\int_{0}^{a} e^{i z s} h(s) d s \in K_{a}
$$

Из (4.16) и последних двух равенств вытекает, что $S$ принадлежит коммутанту $J_{a}$ и является изоморфизмом пространства $L_{2}(0, a)$. В силу формулы $(4.15)$

$$
g(t)=\left(S g_{w}\right)(t)=\frac{d}{d t} \int_{0}^{t} k(t-s) g_{w}(s) d s, \quad 0 \leqslant t \leqslant a .
$$

Доопределим $g$ при $t>a$ этой же формулой и докажем, что $g \in \mathscr{M}$. При $\operatorname{Im} z<0$ с учетом формулы (3.12) имеем

$$
z \int_{0}^{\infty} e^{-i z t} \frac{d}{d t} \int_{0}^{t} k(t-s) g_{w}(s) d s d t=\tilde{s}(-z) w_{-}(z)=s(z) q(z) w_{-}(z)
$$


где $\tilde{s}(-z)=s(z) q(z)-$ внутренне-внешняя факторизация функции из $H_{-}^{\infty}$. Поскольку $|\tilde{s}(x+i 0)| \asymp 1$, то $|q(x-i 0)| \asymp 1$ почти всюду на $\mathbb{R}$. Поэтому для внешней в области $\operatorname{Im} z<0$ функции $w:=q w_{-}$вес $|w(x-i 0)|^{2}$ удовлетворяет условию $\left(\mathrm{A}_{2}\right)$. Заметим еще, что из (4.18) вытекает тождество

$$
s(z)(q(z) p(-z))+e^{-i a z} \psi(-z) \equiv 1, \quad \operatorname{Im} z<0,
$$

которое в силу теоремы о короне [17] равносильно оценке снизу:

$$
|s(z)|+\left|e^{-i a z}\right| \geqslant \delta, \quad \operatorname{Im} z<0
$$

Следовательно, функция, стоящая справа в (4.20), изображается в виде (4.13), т.е. $g \in \mathscr{M}$.

Для доказательства обратного утверждения необходимо рассуждать в обратном порядке. Пусть $g \in \mathscr{M}$, а $s, w$ - отвечающие ей множители в (4.13). По $s(-z) \in H_{+}^{\infty}$ построим оператор $S$ и получим равенство (4.19), в котором $g_{w}$ порождается внешним множителем $w$. Поэтому справедливы равенства $(4.15)$, и если оператор $S$ непрерывно обратим, то квазиэкспонента вида (4.2) изоморфна заведомо регулярной квазиэкспоненте $e_{w}$. Но оператор $S$ обратим потому, что для функции $s(-z)$ сушествуют $p, \psi \in H_{+}^{\infty}$, для которых имеет место тождество $(4.18)$, и стало быть, верно (4.17).

Из теорем 4.4 и 4.5 вытекает

СлЕДСТВИЕ. Пусть $g \in L_{2}(0,2 \pi)$. Тогда семейство функций

$$
\frac{d}{d t} \int_{0}^{t} g(t-s) e^{i n s} d s, \quad n \in \mathbb{Z}
$$

образует безусловный базис пространства $L_{2}(0,2 \pi)$ в том и только том случае, когда $g \in \mathscr{M}_{2 \pi}$.

Полученные результаты дают возможность проиллюстрировать теорему 3.3 о полугруппах на примере оператора $A$, определенного равенством

$$
\left(A^{-1} h\right)(t)=i \int_{0}^{t} h(s) d s+(h, f) g, \quad h \in L_{2}(0, a)
$$

где $f, g$ - пара функций, согласованная с $J_{a}$. Из теорем 4.3 и 4.5 вьводится

Tеорема 4.6. Oператор $A$ вида (4.21) генерирует $L_{2}$-устойчивую полугруппу $U_{t}=\exp \{i A t\}$ тогда и только тогда, когда выполнена совокупность условий:

1) $\sigma(A)$ лежит в области $\operatorname{Im} z>\delta>0$;

2) вес $W^{2}(x)=|\varphi(x)|^{2}\|g(x)\|^{-2}$ удовлетворяет условию $\left(\mathrm{A}_{2}\right)$ на $\mathbb{R}$;

3) $g \in \mathscr{M}_{a}$. 
Используемые здесь обозначения те же, что и в теореме 3.3. Заметим также, что этой формулировке можно придать симметричную форму, указав класс функций, которому должна принадлежать $f$.

Таким образом, из предыдущих результатов вытекает, что важное значение приобретает задача об установлении признаков регулярности произвольной квазиэкспоненты. В основу таких признаков может быть положено любое утверждение, в котором в явной либо в скрытой форме фигурирует понятие регулярной квазиэкспоненты.

Напомним, что регулярные квазиэкспоненты - это в точности те квазиэкспоненты, которые наследуют свойство функции $\exp \{i z t\}$ образовывать безусловные базисы пространств $L_{2}(0, a)$ из своих значений. Но $\exp \{i z t\}$ порождает также и континуальный аналог тригонометрических рядов - преобразование Фурье. Поэтому интересно выяснить, какие квазиэкспоненты наследуют это свойство классических экспонент. Точно вопрос формулируется так: для каких $\mathfrak{H}$-значных квазиэкспонент е существуют константы $m, M>0$ такие, что

$$
m\|h\|^{2} \leqslant \int_{\mathbb{R}}|(e(x), h)|^{2}\|e(x)\|^{-2} d x \leqslant M\|h\|^{2} \quad \forall h \in \mathfrak{H} .
$$

Заметим, что для $L_{2}(0, a)$-значной квазиэкспоненты $\exp \{i x t\}$ неравенства (4.22) превращаются в равенства с $m=M=2 \pi a^{-2}$. Следующий результат имеет принципиальное значение для приложений.

ТЕОРЕМА 4.7. Пусть е - произвольная квазиэкспонента со значениями в пространстве $\mathfrak{H}$, әкспоненциальный тип которой равен а. Тогда следующие условия әквивалентны:

1) двусторонняя оценка (4.22) имеет место для всех $h \in \mathfrak{H}$;

2) квазиәкспонента е регулярна.

Докажем сначала две леммы.

ЛЕмма 4.1. Если квазиэкспонента е удовлетворяет условию (4.22), то справедливы все виводы теоремы 4.1.

ДокАЗАТЕЛЬство. Прежде всего заметим, что

$$
\int_{\mathbb{R}} w^{-2}(x)\left(1+x^{2}\right)^{-1} d x<\infty, \quad w^{2}(x):=\|e(x)\|^{2} .
$$

В самом деле, из условия 1) определения квазиэкспоненты вытекает, что

$$
\begin{aligned}
\int_{\mathbb{R}} w^{-2}(x) x^{-2}|(e(x), e(z))-(e(0), e(z))|^{2} d x \\
\quad=\int_{\mathbb{R}} w^{-2}(x)\left|\left(e(x), h_{z}\right)\right|^{2} d x \leqslant M\left\|h_{z}\right\|^{2}, \quad z \in \mathbb{C} .
\end{aligned}
$$

Поскольку при $\varepsilon>0$

$$
\int_{|x|>\varepsilon} w^{-2}(x) x^{-2}|(e(x), e(z))|^{2} d x \leqslant\|e(z)\|^{2} \int_{|x|>\varepsilon} x^{-2} d x
$$


то имеет место (4.23). Далее, из левого неравенства (4.22) вытекает, что

$$
\mathfrak{H}=\operatorname{clos} \operatorname{span}\{e(z): z \in \mathbb{C}\} .
$$

Снова в силу условия 1) определения 1 для конечных сумм имеем

$$
\left(\tilde{e}(\lambda), \sum c_{k} e\left(z_{k}\right)\right)=\left(e(\lambda), \sum c_{k} h_{z_{k}}\right), \quad \lambda \in \mathbb{C} .
$$

Поэтому линейное отображение

$$
L\left(\sum c_{k} e\left(z_{k}\right)\right):=\sum c_{k} h_{z_{k}}
$$

определено корректно. Из левого неравенства (4.22) для всюду плотного множества векторов $h=\sum c_{k} e\left(\lambda_{k}\right)$ в силу $(4.23)$ найдем

$$
\begin{aligned}
\|L h\|^{2} \leqslant & \frac{1}{m} \int_{\mathbb{R}} w^{-2}(x)|(e(x), L h)|^{2} d x=\frac{1}{m} \int_{\mathbb{R}} w^{-2}(x)|(\tilde{e}(x), h)|^{2} d x \\
\leqslant & \frac{1}{m} \int_{|x| \leqslant \varepsilon} w^{-2}(x)\|\tilde{e}(x)\|^{2} d x\|h\|^{2}+\frac{1}{m} \int_{|x|>\varepsilon} w^{-2}(x) x^{-2}|(e(x), h)|^{2} d x \\
& +\frac{1}{m} \int_{|x|>\varepsilon} w^{-2}(x) x^{-2}|(e(0), h)|^{2} d x \leqslant C\|h\|^{2},
\end{aligned}
$$

т.е. оператор $L$ ограничен. Из определения $L$ вытекает равенство

$$
\tilde{e}(z)=\operatorname{Be}(z), \quad z \in \mathbb{C}, \quad B:=L^{*} .
$$

Поэтому осталось доказать, что $B$ - одноклеточный оператор класса $\Lambda^{(\exp )}$. Докажем сначала, что $B$ принадлежит идеалу Гильберта-Шмидта. В самом деле, для произвольного ортонормированного базиса $\left\{u_{k}\right\}_{-\infty}^{+\infty}$ пространства $\mathfrak{H}$ имеем

$$
\begin{aligned}
\sum_{k=-\infty}^{+\infty}\left\|L u_{k}\right\|^{2} & \leqslant \frac{1}{m} \int_{\mathbb{R}} \sum_{k=-\infty}^{+\infty} w^{-2}(x)\left|\left(B e(x), u_{k}\right)\right|^{2} d x \\
& =\frac{1}{m} \int_{\mathbb{R}} w^{-2}(x) x^{-2} \sum_{k=-\infty}^{+\infty}\left|\left(e(x), u_{k}\right)-\left(e(0), u_{k}\right)\right|^{2} d x \\
& \leqslant M+\frac{2}{m} \int_{|x|>\varepsilon} w^{-2}(x) x^{-2} d x\|e(0)\|^{2}+\frac{2}{m} \int_{|x|>\varepsilon} x^{-2} d x<\infty
\end{aligned}
$$

Чтобы доказать, что у оператора $B$ отсутствует дискретный спектр, заметим, что из (4.24) следует представление

$$
e(z)=(I-z B)^{-1} g, \quad g:=e(0), \quad z^{-1} \notin \sigma(B) .
$$

Раскладывая резольвенту в ряд в окрестности $z=0$, заключаем, что $g$ цикличен для $B$. Пусть теперь $z_{k}$ - собственное число оператора $B, P_{k}$ - соответствующий проектор Рисса. Из последнего равенства выводим

$$
P_{k} \sum c_{j} B^{j} e(z)=(I-z B)^{-1} P_{k} \sum c_{j} B^{j} g
$$

причем слева стоит целая функция, а функция справа такой быть не может в силу конечномерности $P_{k}$. Поэтому $P_{k}=0$ и, стало быть, $z_{k} \notin \sigma(B)$. Теперь доказательство заканчивается точно так, как и доказательство теоремы 4.10. 
ЛЕмма 4.2. Если для квазиэкспоненты е имеет место двусторонняя оценка $(4.22)$, то вес $w^{2}(x):=\|e(x)\|^{2}$ удовлетворяет условию $\left(\mathrm{A}_{2}\right)$ на $\mathbb{R}$.

ДокАЗАТЕльство. Имеем представление (4.25) с одноклеточньм оператором $B \in \Lambda^{(\exp )}$. Из (1.4) вьводим

$$
\int_{\mathbb{R}} x^{-2}\|e(x)-e(0)\|^{2} d x=\int_{\mathbb{R}}\left\|B(I-x B)^{-1} g\right\|^{2} d x \leqslant M\|g\|^{2} .
$$

Вместе с (4.23) это означает, что

$$
\int_{\mathbb{R}} w^{ \pm 2}(x)\left(1+x^{2}\right)^{-1} d x<\infty
$$

Как и в $\oint 1$, вводим внешние функции $w_{ \pm}$. Легко видеть, что для квазиэкспоненты $e$ лемма 1.3 остается в силе. Отметим, что вектор $g=e(0)$ не изображается в виде $g=B u(u \in \mathfrak{H})$. Действительно, в противном случае снова из (1.4) вьводим противоречие:

$$
\left(\int_{\mathbb{R}}(1+|x|)^{-1} d x\right)^{2} \leqslant \int_{\mathbb{R}} w^{-2}(x)(1+|x|)^{-2} d x \int_{\mathbb{R}}\left\|B(1-x B)^{-1} u\right\|^{2} d x<\infty .
$$

Поэтому можно так подобрать $\alpha \in \mathbb{R}$, что пара $\alpha g, g$ будет согласованной с оператором $B$, и, следовательно, по формуле (0.1) она порождает оператор $A$. Этот оператор антидиссипативен $\left(\operatorname{Im}(A f, f) \leqslant 0, f \in D_{A}\right)$, т.е. $\sigma(A)$ лежит в замкнутой нижней полуплоскости. Теперь рассмотрим оператор $A_{z}$ с блуждающей точкой $z$ и будем рассуждать так, как при доказательстве леммы 1.4 , только вместо $\mathbb{R}$ берем прямую $\mathbb{R}+i \varepsilon(\varepsilon>0)$. Мы снова получим неравенство

$$
\int_{\mathbb{R}+i \varepsilon}\|e(\mu)\|^{2}|\mu-z|^{-2} d \mu \leqslant(\pi+M(\operatorname{Im} z-\varepsilon))(\operatorname{Im} z-\varepsilon)^{-1}\|e(z)\|^{2}, \quad \operatorname{Im} z>\varepsilon
$$

с константой $M$, которая не зависит от $\varepsilon$. Поэтому оно сохраняется и при $\varepsilon=0$ :

$$
\int_{\mathbb{R}} w^{2}(x)|x-z|^{-2} d x \leqslant(\pi+M \operatorname{Im} z)(\operatorname{Im} z)^{-1}\|e(z)\|^{2} .
$$

Поскольку лемма 1.3 имеет место, то справедлива оценка

$$
\frac{\operatorname{Im} z}{\pi} \int_{\mathbb{R}} w^{2}(x)|x-z|^{-2} d x \leqslant K\left|w_{+}(z)\right|^{2}, \quad \operatorname{Im} z>0 .
$$

Далее, из (4.26) легко получить оценку снизу для $\|e(z)\|^{2}$. В самом деле, поскольку

$$
\mathbb{P}_{-} \frac{w_{+}(x)}{x-z}=\frac{w_{+}(z)}{x-z}, \quad \operatorname{Im} z>0
$$

где $\mathbb{P}_{-}-$ортопроектор из $L_{2}(\mathbb{R})$ на $H_{-}^{2}$, то

$$
\frac{\pi\left|w_{+}(z)\right|^{2}}{\operatorname{Im} z} \leqslant \int_{\mathbb{R}}\left|w_{+}(x)(x-z)^{-1}\right|^{2} d x \leqslant(\pi+M \operatorname{Im} z)(\operatorname{Im} z)^{-1}\|e(z)\|^{2}
$$


и, следовательно,

$$
\frac{\pi}{\pi+M \operatorname{Im} z}\left|w_{+}(z)\right|^{2} \leqslant\|e(z)\|^{2}, \quad \operatorname{Im} z>0
$$

Из соотношения

$$
\frac{e(z)-e(x)}{z-x}=B(I-z B)^{-1}(I-x B)^{-1} e(0)
$$

и оценок (4.22) выводим

$$
\begin{aligned}
& \int_{\mathbb{R}} w^{-2}(x)\left|\frac{(e(z), h)-(e(x), h)}{z-x}\right|^{2} d x \\
& \quad=\int_{\mathbb{R}} w^{-2}(x)\left|\left(e(x), B^{*}\left(I-\bar{z} B^{*}\right)^{-1} h\right)\right|^{2} d x \\
& \quad \leqslant M\left\|B^{*}\left(I-\bar{z} B^{*}\right)^{-1} h\right\|^{2}=M\left\|\left(\left(B^{*}\right)^{-1}-\bar{z} I\right)^{-1} h\right\|^{2}, \quad h \in \mathfrak{H}, \operatorname{Im} z>0 .
\end{aligned}
$$

Примем теперь во внимание следуюшие два факта. Во-первых, для неограниченного диссипативного оператора $\left(B^{*}\right)^{-1}$ справедлива оценка

$$
\left\|\left(\left(B^{*}\right)^{-1}-\bar{z} I\right)^{-1} h\right\|^{2} \leqslant(\operatorname{Im} z)^{-2}\|h\|^{2}, \quad h \in \mathfrak{H}, \quad \operatorname{Im} z>0 .
$$

Во-вторых, имеет место неравенство

$$
\begin{aligned}
\int_{\mathbb{R}} w^{-2}(x)\left|\frac{(e(x), h)}{z-x}\right|^{2} d x & <\frac{1}{(\operatorname{Im} z)^{2}} \int_{\mathbb{R}} w^{-2}(x)|(e(x), h)|^{2} d x \\
& \leqslant \frac{M}{(\operatorname{Im} z)^{2}}\|h\|^{2}, \quad h \in \mathfrak{H} .
\end{aligned}
$$

Соединяя это с (4.29), найдем

$$
|(e(z), h)|^{2} \int_{\mathbb{R}} w^{-2}(x)|x-z|^{-2} d x \leqslant \frac{K}{(\operatorname{Im} z)^{2}}\|h\|^{2}, \quad h \in \mathfrak{H}, \quad \operatorname{Im} z>0,
$$

что вместе с (4.28) дает

$$
\frac{\pi\left|w_{+}(z)\right|^{2}}{\pi+M \operatorname{Im} z} \int_{\mathbb{R}} w^{-2}(x)|x-z|^{-2} d x \leqslant \frac{k}{(\operatorname{Im} z)^{2}}
$$

или

$$
\frac{\operatorname{Im} z}{\pi} \int_{\mathbb{R}} w^{-2}(x)|x-z|^{-2} d x \leqslant \frac{k}{\pi^{2}} \frac{\pi+M \operatorname{Im} z}{\operatorname{Im} z}\left|w_{+}(z)\right|^{-2} \leqslant K_{1}\left|w_{+}(z)\right|^{-2}
$$

если $\operatorname{Im} z \geqslant \delta>0$. Пусть теперь $0<\operatorname{Im} z \leqslant \delta$. Тогда, рассуждая так же, как и при вьводе (1.5), получим

$$
\left\|\left(\left(B^{*}\right)^{-1}-z I\right)^{-1} h\right\|^{2}=\left\|\int_{0}^{a} e^{-i z t} V_{t}^{*} h d t\right\| \leqslant C_{\delta}\|h\|^{2}, \quad 0<\operatorname{Im} z \leqslant \delta .
$$


Кроме того, имеет место оценка

$$
\int_{\mathbb{R}} w^{-2}(x)\left|\frac{(e(x), h)}{z-x}\right|^{2} d x \leqslant\|h\|^{2} \int_{\mathbb{R}} \frac{d x}{|z-x|^{2}}=\frac{\pi}{\operatorname{Im} z}\|h\|^{2} .
$$

Из (4.29), (4.28) и двух последних оценок следует, что

$$
\frac{\pi\left|w_{+}(z)\right|^{2}}{\pi+M \operatorname{Im} z} \int_{\mathbb{R}} \frac{w^{-2}(x) d x}{|z-x|^{2}} \leqslant \frac{C_{1}}{\operatorname{Im} z},
$$

или

$$
\frac{\operatorname{Im} z}{\pi} \int_{\mathbb{R}} w^{-2}(x)|x-z|^{-2} d x \leqslant K_{2}\left|w_{+}(z)\right|^{-2}, \quad 0<\operatorname{Im} z \leqslant \delta .
$$

Вместе с (4.30) это означает, что интеграл Пуассона для веса $w^{-2}$ допускает оценку, аналогичную (4.27). Поэтому вес $w^{2}$ удовлетворяет условию Макенхаупта.

ДокАЗАТЕльство ТЕОРЕмЫ 4.7. Импликация 1) $\Rightarrow 2$ ). Каждая целая функция $F(z):=(e(z), h)$ удовлетворяет условиям

$$
\int_{\mathbb{R}} w^{-2}(x)|F(x)|^{2} d x<\infty, \quad h_{F}(\pi / 2) \leqslant 0, \quad h_{F}(-\pi / 2) \leqslant a .
$$

Заметим, что если учесть лемму 4.1, то приведенные неравенства для индикатора фактически были установлены при доказательстве леммы 1.3. В силу леммы 4.2 и уже упоминавшегося в $\S 3$ весового аналога теоремы Винера-Пэли имеет место единственное интегральное представление

$$
F(z)=\left(g_{w}(t, z), f\right)_{L_{2}(0, a)}
$$

где ядро $g_{w}(t, z)$ строится по формуле (3.13). Таким образом, имеем линейное отображение $L$ из $\mathfrak{H}$ в пространство $L_{2}(0, a)$ :

$$
(e(z), h)=\left(g_{w}(t, z),(L h)(t)\right)_{L_{2}(0, a)} .
$$

В силу условия теоремы и оценок (3.16) имеем

$$
\begin{aligned}
\|h\|^{2} & \asymp \int_{\mathbb{R}} w^{-2}(x)|(e(x), h)|^{2} d x \\
& =\int_{\mathbb{R}} w^{-2}(x)\left|\left(g_{w}(t, x), L h\right)\right|^{2} d x \asymp\|L h\|^{2}, \quad h \in \mathfrak{H} .
\end{aligned}
$$

Обозначим через $\mathfrak{L}$ образ оператора $L$ в пространстве $L_{2}(0, a)$ и докажем, что $\mathfrak{L}$ инвариантно относительно $J_{a}^{*}$. Если $f \in \mathfrak{L}$, то в силу (4.31) существует такой вектор $h \in \mathfrak{H}$, что

$$
(e(z), h)=\left(g_{w}(t, z), f\right), \quad z \in \mathbb{C} .
$$

Поэтому

$$
\begin{aligned}
\left(g_{w}(t, z), J_{a}^{*} f\right) & =\left(J_{a}\left(I-z J_{a}\right)^{-1} g_{w}, f\right)=\frac{1}{z}\left\{\left(g_{w}(t, z), f\right)-\left(g_{w}(t, 0), f\right)\right\} \\
& =\frac{1}{z}\{(e(z), h)-(e(0), h)\}=(B e(z), h)=\left(e(z), B^{*} h\right),
\end{aligned}
$$


т.е. $J_{a}^{*} f \in \mathfrak{L}$, если $f \in \mathfrak{L}$. Отсюда вытекает, что $\mathfrak{L}=L_{2}(0, a)$ и, стало быть, $L$ - изоморфизм $\mathfrak{H}$ на $L_{2}(0, a)$. В самом деле, если $\mathfrak{L} \varsubsetneqq L_{2}(0, a)$, то оно состоит из функций, почти всюду обрашающихся в нуль на некотором сегменте $[a-\varepsilon, a]$ $(\varepsilon>0)$ [1]. Поэтому в интегральном представлении (4.31) интегрирование всякий раз ведется по сегменту $[0, a-\varepsilon]$ и, значит, тип $e$ меньше $a$, тогда как $a$ и есть тип $e$. Таким образом, из (4.31) выводим, что

$$
e(z)=S g_{w}(z, t), \quad S:=L^{*},
$$

где $g_{w}(z, t)$ есть $w$-квазиэкспонента со значениями в $L_{2}(0, a)$.

Импликация 2$) \Rightarrow 1$ ). В силу теоремы $4.3 e$ изоморфна $w$-квазиэкспоненте $e_{w}$ с $w^{2}(x)=\|e(x)\|^{2}$. Остается заметить, что (4.22) для $w$-квазиэкспонент было установлено в [4].

Как это часто бывает, с континуальњыми аналогами понятий работать легче, чем с дискретными понятиями. Теорема 4.7 положена в основу нескольких, просто формулируемых признаков регулярности квазиэкспонент. На них основаны те приложения, о которых шла речь во введении. Соответствующие результаты предполагается опубликовать в другой статье.

\section{Список литературы}

1. Бродский M. С. Треугольные и жордановы представления линейных операторов. М.: Наука, 1969.

2. Крейн С. Г., Хазан М. И. Дифференциальные уравнения в банаховом пространстве // Итоги науки и техники. Современные проблемы математики. Матем. анализ. Т. 21. М.: ВИНИТИ, 1983. С. 130-264.

3. Губреев Г. М. Спектральньй анализ биортогональных разложений функций в ряды экспонент // Изв. АН СССР. Сер. матем. 1989. Т. 53. №6. С. 1236-1268.

4. Губреев Г. М. Спектралњный анализ биортогональных разложений, порождаемых весами Макенхаупта // Записки научн. сем. ЛОМИ. 1991. Т. 190. С. 34-80.

5. Губреев Г. М. Спектральный анализ биортогональных разложений, порождаемых весами Макенхаупта. Дисс. ... докт. физ.-мат. наук. Харьков: ФТИНТ, 1995.

6. Губреев Г. М. Об одном классе безусловных базисов гильбертовых пространств и о проблеме подобия диссипативных вольтерровых операторов // Матем. сб. 1992. Т. 183. №9. С. $105-146$.

7. Havin V.P., Nikolski N.K. (eds) Linear and complex analysis. Berlin: Springer-Verlag, 1994.

8. Губреев Г. М. Безусловные базисы гильбертовых пространств из значений квазиэкспонент // Сб. трудов, посвящ. 75-летию Одесского гос. пед. ин-та. Одесса, 1994. С. 4-9.

9. Губреев Г. М. Безусловные базисы гильбертовых пространств, составленные из значений целых вектор-функций экспоненциального типа // Функц. анализ и его прилож. 1999. T. 33. № 1. C. $62-65$.

10. Datko $R$. Uniform asymptotic stability of evolutionary processes in Banach space // SIAM J. Math. Anal. 1972. V. 3. P. 428-445.

11. Левин Б. Я. Распределение корней целых функций. М.: ГИТТЛ, 1956.

12. Гарнетт Джс. Ограниченные аналитические функции. М.: Мир, 1984.

13. Hunt R., Muckenhoupt B., Wheeden $R$. Weighted norm inequalities for the conjugate function and Hilbert transform // Trans. Amer. Math. Soc. 1973. V. 176. P. 227-251.

14. Гохберг И. Ц., Крейн М. Г. Введение в теорию линейных несамосопряженных операторов в гильбертовом пространстве. М.: Наука, 1965. 
15. Секефальви-Надь Б., Фойаш Ч. Гармонический анализ операторов в гильбертовом пространстве. М.: Мир, 1970.

16. Минкин A. M. Необходимость условия Макенхаупта для безусловной базисности собственных функций // Алгебра и анализ. 1998. Т. 10. № 3. С. 65-91.

17. Никольский Н. К. Лекции об операторе сдвига. М.: Наука, 1980.

Южно-Украинский педагогический государственншй университет (г. Одесса)

Поступила в редакцию 01.02 .1999 